(Aus dem Institut für Tierphysiologie der landw. Akademie Bonn-Poppelsdorf.)

\title{
Beitrag \\ zur Aufnahme und Verwertung von Kalk und Phosphorsäure durch den Darm.
}

Von

Dr. phil. F. Znckmayer.

(Mit 1 Textfigur.)

Dem Mineralstoffwechsel und besonders dem Kalkstoffwechsel ist in den letzten Jahren in vermehrtem Maasse Aufmerksamkeit geschenkt worden. Aron, Kronheim und Müller, Dibbelt u. A. haben den Kalkstoffwechsel in seinem Zusammenhang mit Erkrankungen der Knochen betrachtet, während Kochmann's Versuche dem Kalkstoffwechsel und dessen Beeinflussung durch Nahrungsmittel gelten. Auf eine Zusammenstellung der Literatur kann ich um so mehr verzichten, als wir in verschiedenen Arbeiten (z. B. Aron 1908) sowohl als auch in Albu-Neuberg's "Mineralstoff-

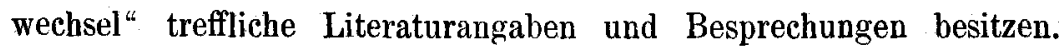
Aus allen diesen Arbeiten geht ebenso wie aus der älteren Literatur die Wichtigkeit des Kalkumsatzes hervor. Die Studien von Kochmann und Petzsch (Biochem. Zeitschr. Bd. 31 Heft 5 und 6, Bd. 32 Heft 1. 1911) beweisen die Abhängigkeit des Kalkstoffwechsels von den organischen Nahrungskomponenten. K o ch m a n n findet einen Mehrverbrauch von Kalk bei vermehrter Nahrung und fasst den Kalkverbrauch des Organismus als einen Vorgang zum Schutze gegen toxische Stoffwechsel-Endprodukte auf. Er verwendet bei seinen Versuchen verschiedene Kalksalze $-\mathrm{CaCl}_{2}$ und $\mathrm{Ca}_{3}\left(\mathrm{PO}_{4}\right)_{2}-$ und findet bei beiden die gleiche Wirkung auf den Kalkumsatz. Es gelingt Kochmann, einerseits mit einer Zulage von anorganischen Kalksalzen eine negative Kalkbilanz in eine positive und andererseits mittels einseitiger kalkfreier Nahrungszulage die positive in eine negative Kalkbilanz zu verwandeln. Nach diesen 
Arbeiten ist also die Aufnahme des Kalkes aus den Nahrungsmitteln noch in anderem Sinne, als man bisher angenommen hatte, wichtig. Es scheint, dass nur dann auf keine Unterbilanz im Kalkumsatz gerechnet werden kann, wenn genügend kalkreiche Nahrung aufgenommen wird.

In Milch und Fleisch bzw. Fleischsaft finden wir den Kalk in Gemeinschaft mit Phosphorsäure zusammen mit Eiweisskörpern in einer durch Alkalikarbonate vicht unter allen Umständen fällbaren Form, was vielleicht $\mathrm{zu}$ der Bezeichnung "in organischer Bindung ${ }^{*}$ Veranlassung gegeben haben mag. Dieser Kalk lässt sich den genannten Nahrungsmitteln mittelst verdünnter Säuren wenigstens zum grössten Teil schon in der Kälte entziehen und zeigt dann alle Fällungsreaktionen der anorganischen Kalksalze. Es besteht also nur so lange ein gewisser Unterschied zwischen anorganischem Kalk und Kalk in sogenannter "organischer Bindung " im Verhalten gegen verdünnte Sodalösung, als die organischen kalkhaltigen Substanzen vollständig unangegriffen, d. h. mit Säuren nicht in Berührung gekommen sind. Wenn aber ein gewisser Unterschied zwischen Nahrungs- und anorganischem Kalk besteht, so ist er in dem Zusammenhang mit organischen Körpern zu suchen. Dass hier vor allem Eiweisskörper oder diesen nahestehende Verbindungen in Frage kommen, lässt sich mit einer gewissen Wahrscheinlichkeit aus dem Verhalten des Milchkalkes schliessen, der wohl eine ganz besondere Verwandtschaft zum Kaseineiweiss besitzt, da er nur sebr schwer vollständig von ihm zu trennen ist. Der beim Ausfällen des Kaseins bei diesem verbleibende und sich mit diesem in Alkali lösende Kalk findet sich niemals ohne gleichzejtige Anwesenheit von Phosphorsäure, wie man bei Versuchen zur Reindarstellung von Kasein beobachten kann.

Gelegentlich verschiedener Arbeiten mit Milcheiweisskörpern schenkte ich dieser Tatsache besondere Beachtung und gelangte so zu einer Reihe von Produkten, welche in der Hauptsache aus Eiweiss bestehen, aber Tricalciumphosphat in bedeutenden Mengen enthalten. Versuche, solche Verbindungen von Kasein mit grösseren Mengen Kalk und Phosphorsäure herzustellen, gelangen unter der Vorsicht der Einhaltung nicht zu stark alkalischer Reaktion und nicht allzu hoher Konzentration. Es konnte durch Behandeln einer Kaseinnatronlösung, die Dinatriumphosphat enthielt, unter Einhaltung der theoretisch notwendigen Menge Alkali (zur Bildung von Trinatrium- 
phosphat und Kaseinnatron) mittelst Chlorcaleium oder durch Einwirkenlassen einer Lösung von Tricalciumphosphat in Salzsäure auf Kaseinnatron unter gewissen Vorsichtsmaassregeln eine stark opaleszente Lösung erhalten werden, die in durchfallendem Lichte völlig klar war. Diese offenbar kolloidale Lösung koagulierte auf Zusatz einer geringen Menge Alkohol, Kochsalz bzw. Ammonsulfat unter Abscheidung eines weissen, wohl filtrierbaren Körpers. Der mit verdünntem Alkohol chlorfrei gewaschene Körper wurde mit Alkohol entwässert und bei niedriger Temperatur getrocknet. Er löste sich auf Zusatz geringer Menge verdünnter Sodalösung, 1/10 Normallauge oder Bikarbonatlösung, zu einer opaleszenten in auffallendem Lichte rötlich gefärbten, in durehfallendem Lichte völlig klaren Flüssigkeit. Seine schwach alkalische Lösung konnte gekocht werden, ohne dass $\mathrm{Ca}_{3}\left(\mathrm{PO}_{4}\right)_{2}$ ausfiel, und setzte auch bei mehrtägigem Stehen bei gewöhnlicher Temperatur keinen Niederschlag ab. Die Untersuchung ergab einen Gehalt von ca. $20 \% \mathrm{Ca}_{3}\left(\mathrm{PO}_{4}\right)_{2}, 101 / 2 \% \mathrm{~N}$ und $10 \%$ Feuchtigkeit. Ich hatte also ein Kasein-Tricalciumphosphat erhalten, das die Eigenschaften des sogenannten organisch gebundenen Kalkes zeigte und mit geringer Menge von Alkalien eine kolloidale Lösung ergab. Der Einfachheit halber will ich dieses Präparat Tricaicol nennen ${ }^{1}$ ).

Um dieses Kalkpräparat zu charakterisieren, wurden Vergleiche mit zentrifugierter Kubmilch angestellt. Das Verhalten beim Kochen mit verdünter Sodalösung war das gleiche; Fällungen wurden bei beiden Produkten nicht erhalten. Dagegen stellte sich heraus, dass Kuhmilch auf Zusatz von Natronlauge bis zur Konzentration einer $\mathrm{N} / 5$-Lauge (45 cem Milch $+5 \mathrm{ccm} Z$ Weifach-Normallauge) in der Kälte nach einiger Zeit, beim Kochen sofort einen Niederschlag gibt, der im wesentlichen aus Calciumphosphat mit einer geringen Menge Eiweiss besteht, ein Verhalten, das auch Handelskasein, welches immer einen gewissen Gehalt an Kalkphosphat aufweist, zeigte. Die Lösung des kolloidalen Kalkphosphatkaseins (Tricalcol) in N/5-Natronlauge ( $4 \% \mathrm{ig}$, also bezüglich ihres Kaseingehaltes ungefähr der Milch gleich) zeigt dieses Verhalten nicht; auch bei tagelangem Stehen bildet sich kein Niederschlag. Hieraus scheint hervorzugehen, dass wenigstens ein Teil des Kalkphosphates der

1) Verfahren zur Herstellung derartiger Produkte, bei denen ausser Kasein auch andere Eiweisskörper Verwendung finden können, ist zum Patent angemeldet. 
Kuhmilch in einer anderen Form vorhanden ist als bei meinem Kalkpräparat. Es lag nun nahe, diesen Versuch auch bei Frauenmilch anzustellen, und ergab sich bei entfetteter Frauenmilch, dass auf Zusatz von Natronlauge bis zur Konzentration N/5 ein Niederschlag auch in der Hitze nicht entstand. Auffälig ist nun, dass die Tricalcollösung mit $0,4 \%$ CaO-Gehalt und Frauenmilch mit ungefähr $0,04 \% \mathrm{CaO}$ keine Fällung ergeben, während Kuhmilch mit ungefähr $0,2 \% \mathrm{CaO}$ einen sehr wesentlichen Niederschlag, der in der Hauptsache aus Kalkphosphat besteht, liefert. Die Entstehung der Fällung kann nicht von der Höhe der Kalkkonzentration abhängen, denn sonst wäre es unerklärlich, dass aus der alkalischen Lösung des Tricalcols trotz ihres doppelt so hohen CaO-Gehaltes das Kalkphosphat nicht ausgeschieden wurde. In der verschiedenen Zusammensetzung der Lösungen konnte dieser Unterschied wohl kaum gesucht werden, besonders da ein entsprechender Zusatz von Milchzucker und Lactalbumin zu der Lösung des kolloidalen Kalkphosphatpräparates "Tricalcol" eine Änderung nicht brachte. Die Erklärung könnte dagegen in einer Verschiedenheit des kolloidalen Zustandes des in der Milch und der Tricalcollösung enthaltenen Kalkphosphates liegen. In dieser Richtung angestellte Versuche haben bis jetzt zu einem Ergebnis nicht geführt.

Was jedoch vor allem wichtig erschien, war die Aufklärung der Frage: Zeigt dieses alkalilösliches Tricalciumphosphat enthaltende Eiweisspräparat "Tricalcol" besondere Verbältnisse bezüglich seiner Resorption? Zur Lösung dieser Frage konnten übliche Stoffwechselversuche nicht dienen, da die Wirkung der sauren Magenverdauung völlig unkontrollierbare Veränderung an dem Präparat hervorbringen konnte. Deshalb wurden verschiedene Resorptionsversuche an abgebundenen Darmschlingen von Kaninchen mit einer schwach alkalischen Lösung des Tricalcol im Vergleiche mit einem Gemische von gewöhnlichem Tricalciumphosphat und Kasein-Natronlösung ausgefuhrt. Das dazu verwendete Kasein war von gleicher Reinheit, wie das zur Darstellung des Tricalcols benutzte. Den Kaninchen wurde nach zwei- bis dreitägigem Hungern in einer möglichst leichten Chloroformnarkose unter aseptischen Kautelen die Bauchhöhle eröffnet und eine mittlere gänzlich leere Dünndarmschlinge hervorgezogen; dann wurden drei Teile derselben von ungefähr je $10-20 \mathrm{~cm}$ Länge mittelst doppelter Ligaturen abgeteilt. In jeden Teil des Darmes wurde nun mittelst einer. Spritze eine 
quantitativ bestimmbare Menge des zu untersucbenden Produktes eingeführt; dann wurde die Bauchwunde entsprechend verschlossen, das Tier nach einiger Zeit getötet und seziert; jeder der drei Darmteile wurde darauf für sich quantitativ ausgespritzt, und in dem so gewonnenen Darminhalt wurde der Kalk bestimmt; die Darmwand selbst wurde gesondert zur Kalkbestimmung benutzt. Es war also bekannt, wieviel Kalk in den entsprechenden Darmteil gebracht. worden war, wieviel davon im Darminhalte und wieviel in der Darmwand selbst vorhanden war. Um sicher zu gehen, wurde dieser Versuch doppelt angestellt. Zur Analyse wurden die Substanzen nach Neumann verascht, der Kalk als Oxalat gefällt und als $\mathrm{CaO}$ gewogen.

Versuch I. Dauer 6 Stunden.

a) In die Darmscblinge von $10 \mathrm{~cm}$ Länge war Tricalcol eingebracht.

Es enthielten: die Eingabe $0,0956 \mathrm{~g}$ und der Darminhalt $0,0848 \mathrm{~g} \mathrm{CaO}$; mithin waren $0,0108 \mathrm{~g} \mathrm{CaO}$ resorbiert.

Das $10 \mathrm{~cm}$ lange Stück Darm enthielt $0,0100 \mathrm{~g} \mathrm{CaO}$.

b) Die zweite, $15 \mathrm{~cm}$ lange Darmschlinge wurde mit einer. Lösung von kalkfreiem Kasein-Natron beschickt.

Der Darminhalt wies trotz der kalkfreien Eingabe einen Gehalt $0,0060 \mathrm{~g} \mathrm{CaO}$ auf.

Die Darmwand des $15 \mathrm{~cm}$ langen Stückes Darn enthielt 0,0052 g CaO.

c) In die dritte, $20 \mathrm{~cm}$ lange Darmschlinge war Tricalciumphosphat feinst suspendiert in einer Lösung von Kasein-Natron eingegeben worden.

Es enthielten $\mathrm{CaO}$ in Gramm: Eingabe $=0,0622 \mathrm{~g}$, Darminhalt $=0,0610 \mathrm{~g}$; es waren also nur $0,0012 \mathrm{~g} \mathrm{CaO}$ resorbiert worden.

Die $20 \mathrm{~cm}$ lange Iarmschlinge enthielt $0,0102 \mathrm{~g} \mathrm{CaO}$. Die Gewinnung des Darminhaltes geschah bei a, b und c durch Abspülen mit $50 \mathrm{ccm}$ destilliertem Wasser.

Bei Wiederholung des Versuches wurde besonders darauf geachtet, dass die Darmenden möglichst gleich lang waren. Die Ligaturen trennten drei genau $10 \mathrm{~cm}$ lange Darmschlingen voneinander. Die Dauer des Versuches betrug 12 Stunden. Die Darmabschnitte wurden genau wie bei Versuch I verarbeitet. Die Analyse ergab folgendes Resultat: 


\section{Versuch II. Dauer 12 Stunden.}

a) Die erste Darmschlinge enthielt Tricalcol in 10\% iger Lösung. Es enthielten $\mathrm{CaO}$ in Gramm: Eingabe $=0,0588 \mathrm{~g}$, Darminhalt $=0,0574 \mathrm{~g}$; mithin waren resorbiert $0,0014 \mathrm{~g} \mathrm{CaO}$.

Die $10 \mathrm{~cm}$ lange Darmwand enthielt $0,0043 \mathrm{~g} \mathrm{CaO}$.

b) Zweite Darmschlinge: blieb leer. Dieser Darmabschnitt wurde nach dem Ausspülen verascht. Er enthielt 0,0063 g CaO.

c) In die dritte Darmschlinge wurde Tricalciumphosphat suspendiert in Kasein-Natronlösung eingegeben. Die Eingabe entsprach $0,0596 \mathrm{~g} \mathrm{CaO}$, während der Darminhalt $0,0686 \mathrm{~g} \mathrm{CaO}$ enthielt. Fs waren demnach aus der Darmschlinge $0,0090 \mathrm{~g}$ CaO mehr ausgespült, als eingegeben worden war.

Die $10 \mathrm{~cm}$ lange Darmwand enthielt $0,0036 \mathrm{~g} \mathrm{CaO}$.

Bei diesem Versuch wurde die Gewinnung des Darminhaltes durch Ausspülen mit $150 \mathrm{ccm}$ destilliertem Wasser erreicht, wodurch die unter Darminhalt angegebenen Mengen $\mathrm{CaO}$ im Vergleich mit Versuch I viel höher sind und die Zablen der Resorption niedriger wurden. Dass in Versuch II trotz der längeren Zeit geringere Werte für die Resorption gefunden wurden als in Versuch I, mag wohl von der Verschiedenheit des durch den Operationseingriff hervorgerufenen Allgemeinzustandes herrühren.

Wenn auch vorstehende Zahlen auf eine Aufnahme des Kalkes aus dem Tricalcol schliessen lassen, während für Tricalciumphosphat eine solche Deutung nicht möglich ist, so sind doch die gewonnenen Zahlen so klein, dass sie als vollwertiger Beweis nicht betrachtet werden können. Es wurde daher versucht, die Resorbierbarkeit des kolloidalen Kalkpräparates durch den Darm mit Hilfe einer VellaFistel zu ermitteln ${ }^{x}$.

Einem ca. $8 \mathrm{~kg}$ schweren männlichen 2-3 jährigen Hunde wurde eine mittlere Dünndarmschlinge von ca. $1 \mathrm{~m}$ Länge in die Bauchwand eingeheilt. Herr Privatdozent Dr. O. Prym in Bonn hatte die Güte, die Operation auszuführen; und ich erlaube mir hier nochmals meinen verbindlichsten Dank für die gütige Unterstützung auszusprechen. Die verwendeten und eingeheilten Darmkanülen

1) Herrn Prof. Kochmann, Greifswald, dem ich die Anregung zu diesem Versuche verdanke, erlaube ich mir hier nochmals auch für seine gütigen Ratschläge, die sich mir sehr nützlich erwiesen, verbindlichst zu danken. 
sassen ca. $15 \mathrm{~cm}$ auseinander. Der Hund erholte sich rasch, erhielt reine Fleischnahrung und nahm dabei an Gewicht zu.

Das lebhafte Tier (Fox) war im Anfange nur sehr schwer zu verwenden, um so mehr, als die Wunden nicht ganz glatt verheilt waren. Nach und nach jedoch wurde das Tier ruhiger, die Wunden schlossen sich, und es konnte mit der Arbeit begonnen werden.

Zuerst sollte festgestellt werden, ob Tricalcol resorbierbar ist. $\mathrm{Zu}$ diesem Zwecke wurden $2 \mathrm{~g}$ Tricalcol mit $20 \mathrm{ccm} 0,5 \%$-Sodalösung bei $40^{\circ} \mathrm{C}$. gelöst und diese Lösung mit physiologischer Kochsalzlösung auf $250 \mathrm{ccm}$ verdünnt. Diese auf $38^{\circ} \mathrm{C}$. erwärmte Lösung wurde mittelst eines $6 \mathrm{~mm}$ dicken Katheters, der ungefähr $10 \mathrm{~cm}$ tief in den Darm eingeführt und durch einen darüber geschobenen, umgekrempelten Gummischlauch auf der Darmkanüle befestigt war, innerhalb 3-4 Stunden einlaufen gelassen. Die während des Einlaufens aus dem anderen Ende der Darmschlinge ausfliessende Flüssigkeit wurde durch einen auf der anderen Darmkanüle befestigten Gummischlauch in einem Becherglase aufgefangen. Nach Beendigung des Einlaufenlassens wurde mit 350-400 ccm körperwarmer physiologischer Kochsalzlösung der Darm völlig rein ausgespült, und diese Flüssigkeit wurde mit der während des Versuches aufgefangenen vereinigt und analysiert. Am nächsten Morgen wurde der Darm mit $200 \mathrm{ccm}$ physiologischer Kochsalzlösung gespült und in dieser Fiüssigkeit, die bei ungenügender Ausspülung nach dem Versuche noch wesentliche Mengen Kalk enthalten konnte, der Kalkgehalt bestimmt. Die bei den Vorarbeiten gemachten Erfahrungen zeigten, dass in diesen Spülwässern meist nur geringe Mengen Kalk vorhanden waren, also offenbar durch eine reichliche Ausspülung nach dem Versuche aller im Darm vorhandener Kalk gewonnen wird. Nachdem die ersten Versuche mit dem kolloidalen Kalkpräparat durchgeführt waren, wurde in Kasein-Natronlösung suspendiertes Tricalciumphosphat, und auch in Wasser suspendiertes Tricalciumphosphat in beiden Fällen feinst nass zerrieben, verwendet. Ich führe im folgenden weder die ersten orientierenden noch diejenigen Versuche auf, bei denen durch irgendeine äussere Ursache die Arbeit nicht völlig quantitativ durchgeführt werden konnte. Die angewendeten Mengen der Kalkverbindungen wurden so gross gewählt, dass mit aller Sicherheit die Analysen ausgeführt werden konnten. Die Substanzen wurden nach dem Eindampfen nach N e uman n verascht, der Kalk als Oxalat gefällt und 
nach vorherigem Umfällen als $\mathrm{CaO}$ gewogen. Nachstehende Tabelle gibt über den Verlauf der Versuche Aufschluss.

\begin{tabular}{|c|c|c|c|c|c|c|c|}
\hline \multirow{2}{*}{$\begin{array}{l}\text { Ver- } \\
\text { such } \\
\text { Nr. }\end{array}$} & \multirow{2}{*}{$\begin{array}{l}\text { Dauer } \\
\text { in } \\
\text { Stunden }\end{array}$} & \multirow{2}{*}{$\begin{array}{l}\text { Gelöst } \\
\text { in } \\
\mathrm{ccm}\end{array}$} & \multicolumn{2}{|c|}{ Angewandt } & \multirow{2}{*}{$\begin{array}{l}\text { Aus- } \\
\text { gespültt } \\
\mathrm{CaO} \\
\mathrm{g}\end{array}$} & \multicolumn{2}{|c|}{ Resorbiert } \\
\hline & & & Präparat & $\mathrm{CaO} \mathrm{g}$ & & $\mathrm{CaO} \mathrm{g}$ & $\mathrm{CaO} \%$ \\
\hline $\begin{array}{l}\text { II } \\
\text { III } \\
\text { IV }\end{array}$ & $\begin{array}{l}3 \\
2^{3 / 4} \\
3 \\
2^{1 / 2}\end{array}$ & $\begin{array}{l}250 \\
250 \\
100 \\
250\end{array}$ & $\begin{array}{c}\text { Tricalcol } \\
" \\
"\end{array}$ & $\begin{array}{l}0,2200 \\
0,2200 \\
0,1430 \\
0,2200\end{array}$ & $\begin{array}{l}0,1418 \\
0,1336 \\
0,1173 \\
0,1404\end{array}$ & $\begin{array}{l}0,0782 \\
0,0864 \\
0,0257 \\
0,0796\end{array}$ & $\begin{array}{l}35,5 \\
39,2 \\
18,0 \\
36,2\end{array}$ \\
\hline $\mathrm{V}$ & $2^{1 / 2}$ & 250 & $\begin{array}{l}\mathrm{Ca}_{3}\left(\mathrm{PO}_{4}\right)+\mathrm{Ka}- \\
\text { sein-Natron }\end{array}$ & 0,2004 & 0,2054 & $-0,0050$ & $-2,4$ \\
\hline VI & $2^{1 / 4}$ & 100 & $\mathrm{Ca}_{3}\left(\mathrm{PO}_{4}\right)_{2}$ & 0,2004 & 0,1985 & 0,0019 & 0,9 \\
\hline
\end{tabular}

Während die Versuche I-IV zeigen, dass der Kalk des Tricalcols in grösserer Menge resorbiert wird, ergeben die Versuche $\mathrm{V}$ und VI, dass weder eine Suspension von gewöbnlichem $\mathrm{Ca}_{8}\left(\mathrm{PO}_{4}\right)_{2}$ in KaseinNatronlösung noch eine solche von nass zerriebenem mit physiologischer Kochsalzlösung aufgeschlemmten Tricalciumphosphat eine Verminderung ihres Kalkgehaltes bei der Passage durch den Darm erfahren.

Die bei dieser Versuchsreihe ermittelten Zahlen bestätigen die Versuche an den Kaninchen und beweisen, dass aus einer kolloidalen Kalkphosphat-Kaseinlösung (Tricalcol) durch den Darm Kalk in wesentlicher Menge aufgenommen wird, während dies bei Anwendung des gewöhnlichen Tricalciumphosphats mit oder ohne gleichzeitige Anwesenheit von Kasein nicht der Fall ist. Gleichzeitig ergibt sich, dass die Gegenwart von Kasein eine Aufnahme von gewöhnlichem ungelösten $\mathrm{Ca}_{3}\left(\mathrm{PO}_{4}\right)_{2}$ im Darme nicht bewirkt. Auf die anderen aus diesen Versuchen zu ziehenden Schlüsse komme ich später zurück.

Es interessiert nun weiter, ob der Organismus den resorbierten kolloidalen Kalk verwertet. Hierüber mussten Stoffwechselversuche in Vereinigung mit Resorptionsversuchen am Vella-Fistelhund Aufklärung bringen. Um eine möglichst lange Versuchszeit, Gleichmässigkeit der Versuche und sicheres quantitatives Arbeiten für die Resorptionsversuche erreichen zu können, musste dafür Sorge getragen werden, dass das zu verwendende Tier mehrere Stunden lang täglich völlig ruhig gestellt werden konnte. Es wurde dies in der Weise bewirkt, dass für das Tier ein passendes Geschirr hergestellt wurde, mit dessen Hilfe es an einem Gerüst festgelegt werden konnte. Nach verschiedenen Veränderungen ergab das aus der Figur ersichtliche Geschirr ein völlig zufriedenstellendes Resultat. Das Tier konnte grössere Bewegungen weder vorwärts noch rück- 
wärts, weder in die Höhe noch zum Borlen oder auf die Seite ausführen. Die Füsse wurden noch mit einem breiten Zeugstreifen leicht fixiert, so dass eine nahezu völlige Ruhigstellung ohne Belästigung des Tieres erreicht wurde. Der Hund schwebte in dem Geschirr, wenn er des Stehens müde war; von einer Unbequemlichkeit konnte keine Rede sein, denn das Tier schlief oft stundenlang. während der Resorptionsversuche. Um allen Verlusten vorzubeugen, wurde das Tier auf einen mit Gefälle und Ablauf versehenen Zink-

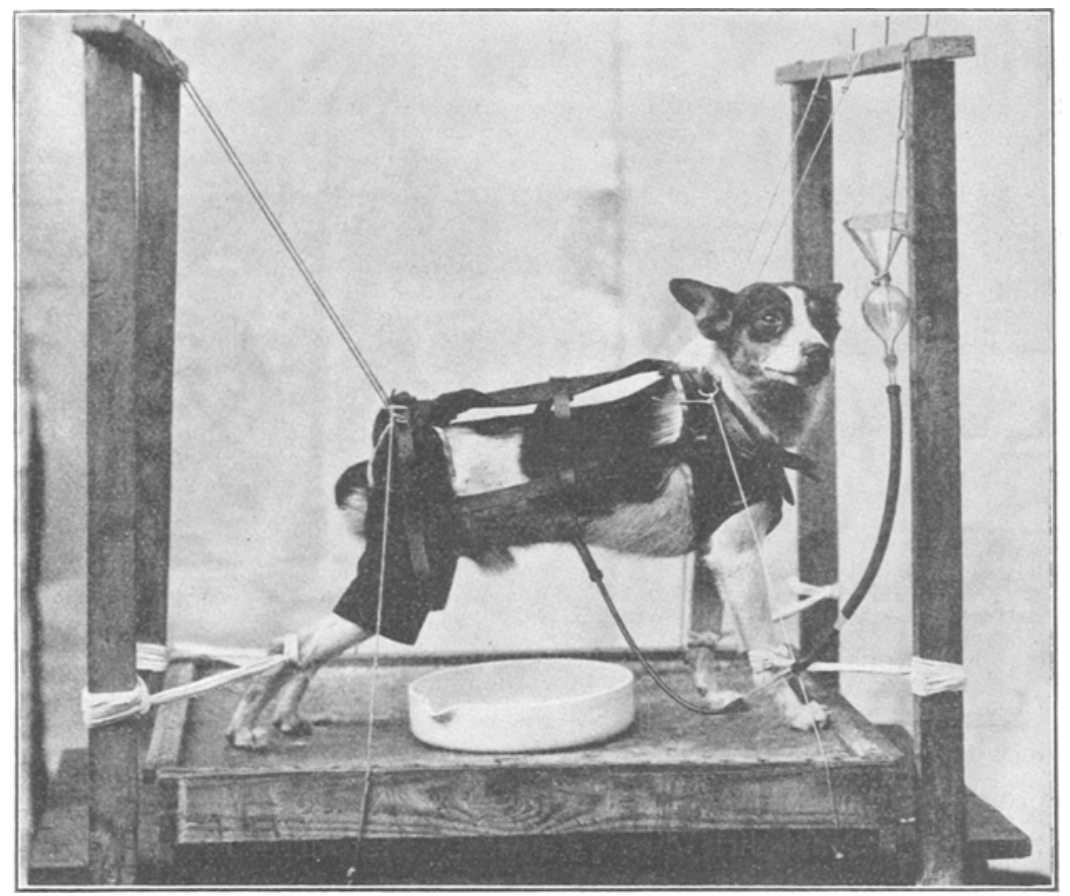

kasten gestellt. Wenn während der Versuche Harn gelassen wäre, was allerdings in der ganzen Versuchsreihe nicht der Fall war, dann konnte derselbe, ohne mit den Analysensubstanzen aus dem Darme in Berührung zu kommen, aufgefangen werden. Etwas erhöht stand unter dem Tiere eine Porzellanschale, die zur Aufnahme der aus der Fistel austretenden Flüssigkeit und zum Sammeln des Spülwassers diente. Es war nämlich nach einiger Zeit die hintere Darmkanüle ausgeheilt, so dass sich zu Beginn dieser Versuche das Darmlumen als ein $2-3 \mathrm{~mm}$ grosses Loch manifestierte. Pf] ũger's Archiv für Physiologie. Bd. 148. 16 
Eine wesentliche Beeinflussung der Arbeitsweise fand hierdurch nicht statt.

Das Tier war in der Zwischenzeit täglich mit der gleichen Menge Pferdefleisch und kalkfreiem Wasser gefüttert und sein isolierter Darmteil täglich mit $250-350$ ccm körperwarmer physiologischer Kochsalzlösung ausgespült worden. In dieser Zeit wurde häufiger die von mehreren Tagen stammende Spülflüssigkeit analysiert, und es ergab sich aus fünf Bestimmungen, dass täglich 2, maximal $3 \mathrm{mg} \mathrm{CaO}$ aus der $1 \mathrm{~m}$ langen Darmsehlinge ausgespült werden konnten. Diese Zahlen scheinen die tatsächlichen Ausscheidungsverhältnisse in dem Darmteile wiederzugeben, da Verluste durch Auslaufen von Daruflussigkeit nicht vorkamen.

Ehe mit dieser Versuchsreihe begonnen wurde, erhielt das Tier während 14 Tagen Versuchsfutter; es lebte in einem mit Zink ausgeschlagenen und mit verzinktem Rost verseheuen, sehr geräumigen Stoffwechselkäfig und war beim Beginn der Versuche vollständig eingewöhnt. Von 6 zu 6 Taqen wurden zur Abgrenzung des Kotes Gummistückchen mit dein Futter gereicht, so dass bezäglich der späteren Trennung des Kotes sich Schwierigkeiten nicht ergeben konnten. Das Futter bestand aus fein gehacktem, wenig fettem Pferdefleisch, das für die ganzen-Versuche zusammen gekauft und in Glasgefässen mit Deckel und Schraubverschluss zu je $490 \mathrm{~g}^{1}$ ) gewogen und gemeinsam sterilisiert wurde. Die tägliche Nahrung betrug also $245 \mathrm{~g}$ sterilisiertes Pferdefleisch, während zum Trinken eine $0,3 \%$ ige Lösung kalkfreien Kochsalzes in destilliertem Wasser gereicht wurde. Das Tier wog zu Beginn des Versuches $8,3 \mathrm{~kg}$ und behielt anfänglich dieses Gewicht. An diese 14 tägige Vorperiode schloss sich ein Stoffwechselversuch von 6 Tagen an. Die Aburenzung des Kotes wurde mittelst sechs bis acht, vorher mit Salzsäure ausgekochten Gummistücken, für jede Periode in verschiedener Farbe, bewerkstelligt. Die Abtrennung gelang scharf, da der Kot yeuügend fest war.

I)ie Aualyse des Futters wurde mehrere Male in Proben, die verschiedenen Glasgefässen entstammten, ausgeführt und erqab gut

1) Es sollten je $250 \mathrm{~g}$ pro Tag verfüttert, also 500-g-Portionen abgewogen werden, aber dann hätte der Vorrat nicht ausgereicht, weshalb nur $490 \mathrm{~g} \mathrm{ab}-$ gewogen wurdetı. 
Beitrag zur Aufnahme und Verwertung von Kalk und Phosphorsäure etc. 235

übereinstimmende Werte. Der Stickstoft wurde nach Kjeldabl bestimmt und die Mineralanalysen wurden nach dem Veraschen und Schmelzen mit Soda und Salpeter vorgenommen. Für die Kalkbestimmung mussten ungefähr $125-150 \mathrm{~g}$ verwandt werden, für die Ermittelung der Phosphorsäure wurden $30 \mathrm{~g}$ Substanz benutzt. Der Kalk wurde als Oxalat gefällt, der oxalsaure Kalk einer Umfällung unterworfen und dann als $\mathrm{CaO}$ gewogen. Die Phosphorsäure wurde aus der gelösten Soda-Salpeterschmelze mit Ammonmolybdat gefällt, in Ammonmagnesiumphosphat übergeführt und als Magnesiumpyrophospbat gewogen. In gleicher Weise wurde anfangs mit dem Harn und Kot verfahren, es ergab sich jedoch, dass die Veraschung nach Neumann bei gleichen Resultaten schneller zum Ziele führte, weshalb später nur nach dieser Methode gearbeitet wurde. Die zur Bestimmung des Kalkes verwendeten Mengen betrugen für Harn $250-300 \mathrm{~g}$, für Kot 3-4 g lufttrocken. Zur Phosphorsäureanalyse wurden $25 \mathrm{~g}$ Harn und $3-4 \mathrm{~g}$ lufttrockener Kot benutzt. Zu den Stickstoffanalysen wurden die Mengen so gewählt, dass jeweils zwischen 50 und $100 \mathrm{ccm}$ Fünftelnormalsäure zur Absättigung nötig waren.

Die Analyse des Futters ergab in Prozenten : $3,06 \mathrm{~N}, 0,0252 \mathrm{CaO}$, $0,3632 \mathrm{P}_{2} \mathrm{O}_{5}$.

Nachstehende Tabelle ergibt die nötigen Daten.

Tabelle I.

\begin{tabular}{|c|c|c|c|c|c|c|c|}
\hline & \multicolumn{2}{|c|}{$\begin{array}{c}\text { Einnahme } \\
\mathbf{g}\end{array}$} & \multirow{2}{*}{$\begin{array}{l}\text { Gesamt- } \\
\text { aufnahme }\end{array}$} & \multicolumn{2}{|c|}{$\begin{array}{c}\text { Ausgabe } \\
\mathrm{g}\end{array}$} & \multirow{2}{*}{$\begin{array}{l}\text { Gesamt- } \\
\text { ausgabe }\end{array}$} & \multirow{2}{*}{$\begin{array}{c}\text { Bilanz } \\
\mathrm{g}\end{array}$} \\
\hline & Futter & $\begin{array}{c}\text { Tri- } \\
\text { calcol }\end{array}$ & & Harn & Kot & & \\
\hline $\begin{array}{l}\mathrm{N} \\
\mathrm{CaO}_{2}:\end{array}$ & $\begin{array}{r}44,9820 \\
0,3705 \\
5,3391\end{array}$ & E & $\begin{array}{r}44,9820 \\
0,3705 \\
5,3391\end{array}$ & $\begin{array}{r}44,8603 \\
0,1868 \\
5,4294\end{array}$ & $\begin{array}{l}2,0395 \\
0,3273 \\
0,3002\end{array}$ & $\begin{array}{r}46,8998 \\
0,5141 \\
5,7296\end{array}$ & $\begin{array}{r}-1,9178 \\
-0,1436 \\
-0,3905\end{array}$ \\
\hline
\end{tabular}

Das Kotgewicht betrug lufttrocken $28,7926 \mathrm{~g}$.

Der Versuch zeigt, dass die Verdaunng des Futters eine ausserorientlich gute, ca. $95,4 \%$ des zugeführten Stickstoffes, war. Die N-Bilanz war negativ, ebenso die Kalk- und Phosphorsäurebilanz. Die ausgeschiedene Phosphorsäuremenge ist grösser, als der Zufuhr entspricht, was durch die Einschmelzung des Körpergewebes seine Erklärung findet. Die mit dem Kot ausgeschiedenen Phosphorsäureund Kalkmengen lassen erkennen, dass diese Substanzen als Tricalciumphosphat ausgeschieden wurden. 
Dieser Periode schloss sich ein Versuch mit genau der gleichen Futtermenge bei Zufuhr von Tricaleol durch den Darm an., In dieser Periode II wurden täglich dem mit dem oben beschriebenen Geschirr in dem Gestell befestigten Hunde $3 \mathrm{~g}$, in der ganzen Periode also $18 \mathrm{~g}$ Tricalcol in einer $10 \%$ igen schwach alkalischen Lösung durch einen Katheter, der in der oben beschriebenen Weise an der Darmkanüle befestigt war, innerhalb einer halben Stunde eingebracht und dann während 5 Stunden in gleichen Zeitabschnitten $100 \mathrm{ccm}$ einer physiologischen Kochsalzlösung, die $0,1 \%$ Soda enthielt, in jedesmaligen Mengen von $10 \mathrm{ccm}$ in den isolierten Darmteil eingegeben. Fs wurde in dieser starken Konzentration gearbeitet, damit alles zugeführte Präparat in dem Darme verblieb und nicht wieder zur hinteren Fistel herausfloss; aus dem gleichen Grunde wurde auch die Menge der physiologischen Kochsalzlösung so bemessen, dass sie in der Versuchszeit resorbiert werden konnte. Eine alkalische Kochsalzlösung zu verwenden, erschien mir wegen Aufrechterhaltung der Alkalität des Darminhaltes notwendig, da ich bei verschiedenen Vorversuchen die im isolierten Darmteile vorhandene bzw. mit physiologischer Kochsalzlösung ausspülbare Menge Alkali sehr verschieden gross fand und manchmal auf recht geringe Mengen Alkali stiess. Eine Stunde nach der Eingabe der alkalischen Kochsalzlösung wurde der Katheter unter Verhütung von Verlusten herausgezogen, die Silberkanüle nebst Umgebung sowie der Katheter gereinigt und darauf die Darmkanüle durch eine festsitzende Gummikappe verschlossen. Ich überzeugte mich oftmals, dass weder die Fistel Flüssigkeit durchliess, noch solche zwischen Bauchwand und Kanile durchdrang. Der Hund wurde nun wieder in den Stoffwechselkäfig gebracht, wo er bis zum nächsten Morgen blieb. Damit das Tier an der verschlossenen Fistel weder beissen noch lecken konnte; trug er fortwährend einen engen Maulkorb. Im Käfig waren niemals auch nur Spuren des Darminhaltes oder Schleimes zu finden, es hatte also ein Ausfluss aus den Fisteln nicht stattgefunden.

Die Fütterung fand täglich in zwei Abteilungen vor Beginn der Vell a-Fistelfullung und nach Beendigung derselben statt; abends wurde das Tier mit kalkfreiem Kochsalzwasser getränkt; am nächsten Morgen kam es ins Geschirr und auf das Gerüst. Zuerst wurde dann die Gummikappe von der Silberkanüle entfernt, der hintere, sehr enge Fistelausgang gereinigt und darauf der Darm mit ca. $300 \mathrm{cem}$ physiologischer Kochsalzlösung von Körpertemperatur aus- 
gespült. Alle diese Operationen wurden entsprechend den Anforderungen an eine quantitative Analyse durchgeführt. Bevor mit der Eingabe der täglichen Dosis der alkalischen Tricalcollösung begonnen werden konnte, wurde eine halbe Stunde nach der Ausspülung gewartet, damit der Darm sich nach Möglichkeit entleeren bzw. die in dem Darm verbliebenen Reste der Kochsalzlösung resorbiert werden konnten. Alle Spül- und Waschwässer wurden gesammelt und täglich auf dem Wasserbade eingedampft. Wie im vorstehenden beschrieben, wurde 6 Tage lang verfahren und in dieser Zeit sechsmal $3=18 \mathrm{~g}$ Tricalcol eingegeben. Die am Tage nach der letzten Eingabe erhaltenen Spülwässer - es wurde aus Vorsicht mit 700 ccm physiologischer Kochsalzlösung ausgespült - wurden mit den vorher gewonnenen Spülwässern vereinigt. Das Befinden des Hundes während dieser Periode war ein durchaus gutes, das Tier zeigte hier eher eine grössere Fresslust, als eine geringere, gegenüber der vorhergehenden Periode. Die Schwierigkeiten eines sauberen quantitativen Arbeitens am Tier sind erhebliche, doch gelangeri alle Operationen aufs beste. Ich möchte noch bemerken, dass sich aus der in dem isolierten Darmteile eingegebenen konzentrierten Lösung des Tricalcols anfänglich grobe Flocken, offenbar Eiweiss, abschieden, die aber im Verlaufe des Versuches wieder verschwanden, so dass bein allmorgendlichen Ausspülen nur in der Silberkanüle einige Flocken des Präparates gefunden werden konnten. Die Spülwässer zeigten eine leicht gelbliche Färbung und nur ganz geringe Trübung; einige Schleimknöllchen waren immer darin enthalten.

Die eingedampften Spülwässer wurden zur Analyse mit Soda und Salpeter geschmolzen, die Schmelze mit Salpetersäure gelöst und in aliquoten Teilen derselben Kalk und Phosphorsäure bestimmt. Von einer N-Bestimmung musste abgesehen werden, da das Material nicht gleichmässig zu mischen und auch nicht in Lösung zu bringen war, ohne dass die Genauigkeit der für mich wichtigeren Kalk- und Phosphorsäurebestimmung darunter gelitten hätte. Von einer Bestimmung des Stickstoffgehaltes konnte um so mehr Abstand genommen werden, als die durch das Tricalcol einverleibte Menge $\mathrm{N}$ im Verhältnis zum Nahrungsstickstoff sehr gering, ungefähr 1,5 zu 45 bzw. 56 war und ausserdem der Stickstoffgehalt, ohne das Endresultat wesentlich $\mathrm{zu}$ beeinflussen, aus der aufgenommenen Menge Kalkphosphat berechnet werden konnte. Tabelle II zeigt die Aufnahme des Tricalcols durch den isolierten Darmteil, während die Tabelle III über den Stoffwechselversuch Aufschluss gibt. 
T abelle II.

Aufnahme des Tricalcols durch die isolierte Darmschlinge in Periode II.

\begin{tabular}{|c|c|c|c|c|}
\hline & $\begin{array}{c}\text { Eingegeben } \\
\mathrm{g}\end{array}$ & $\begin{array}{l}\text { Zurück- } \\
\text { analysiert } \\
\mathrm{g}\end{array}$ & $\begin{array}{c}\text { Resorbiert } \\
\mathbf{g}\end{array}$ & $\begin{array}{l}\text { Resorption } \\
\text { in Prozenten } \\
\text { der Eingabe }\end{array}$ \\
\hline 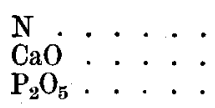 & $\begin{array}{l}1,8450 \\
1,9764 \\
1,6110\end{array}$ & $\begin{array}{c}\text { (geschätzt) } \\
0,6125 \\
0,5439\end{array}$ & $\begin{array}{c}(1,2794) \\
1,3639 \\
1,0671\end{array}$ & $\begin{array}{l}(69,01) \\
69,01 \\
66,24\end{array}$ \\
\hline
\end{tabular}

Tabelle III.

Periode II. 6 Tage. Futter dreimal $490 \mathrm{~g}$ sterilisiertes Pferdefieisch. $18 \mathrm{~g}$ Tricalcol durch die isolierte Darmschlinge.

\begin{tabular}{|c|c|c|c|c|c|c|c|}
\hline & \multicolumn{2}{|c|}{$\underset{\mathrm{g}}{\text { Einnahme }}$} & \multirow{2}{*}{$\begin{array}{l}\text { Gesamt- } \\
\text { aufnahme }\end{array}$} & \multicolumn{2}{|c|}{$\begin{array}{l}\text { Ausgabe } \\
\mathbf{g}\end{array}$} & \multirow{2}{*}{$\begin{array}{l}\text { Gesamt- } \\
\text { ausgabe }\end{array}$} & \multirow{2}{*}{$\begin{array}{c}\text { Bilanz } \\
\mathrm{g}\end{array}$} \\
\hline & Futter & Tricalcol & & Harn & Kot & & \\
\hline $\begin{array}{l}\mathrm{N} \\
\mathrm{CaO} \\
\mathrm{P}_{2} \mathrm{O}_{5}\end{array}$ & $\begin{array}{r}44,9820 \\
0,3705 \\
5,3391\end{array}$ & $\begin{array}{l}1,2794 \\
1,3639 \\
1,0671\end{array}$ & $\begin{array}{r}46,2614 \\
1,7344 \\
6,4062\end{array}$ & $\begin{array}{r}44,9168 \\
0,2760 \\
5,3459\end{array}$ & $\begin{array}{l}2,2048 \\
0,4279 \\
0,3742\end{array}$ & $\begin{array}{r}47,1216 \\
0,7039 \\
5,7201\end{array}$ & $\begin{array}{l}-0,8602 \\
+1,0305 \\
+0,6861\end{array}$ \\
\hline
\end{tabular}

Kotgewicht: lufttrocken $28,4966 \mathrm{~g}$.

Wir sehen aus vorstehender Tabelle, dass die Stickstoff bilanz weniger negativ geworden ist; die Kalkbilanz dagegen ist stark positiv geworden, und auch Phosphorsäure wurde angelagert. Die Besserung der N-Bilanz ist in dieser Periode also bei gleicher Fütterung und Tricalcolzulage beträchtlich; die Verwertung des Stickstoffes - beträgt ungefähr $95,2 \%$. Die Kalkbilanz ist stark positiv geworden, und zwar ist nahezu die ganze Menge des zugeführten Kalkes zur Verwertung gelangt. Dem in Versuch I und II gereichten Futter entspricht, wie aus Periode I hervorgeht, eine negative $\mathrm{CaO}$ - Bilanz von $-0,1436 \mathrm{~g} \mathrm{CaO}$; es wurden nun durch die isolierte Darmschlinge $+1,3639 \mathrm{~g} \mathrm{CaO}$ resorbiert, wodurch die dem Futter entsprechende, um 0,1436 g CaO negative CaO-Bilanz - sich um $+1,1741 \mathrm{~g} \mathrm{CaO}$ auf $+1,0305 \mathrm{~g} \mathrm{CaO}$ erhöhte. Von den aufgenommenen 1,3639 $\mathrm{g} \mathrm{CaO}$ wurden $1,1719 \mathrm{~g} \mathrm{CaO}$, also ungefähr $86 \%$ verwertet, wodurch bewiesen ist, dass der durch den Dünndarm aufgenommene Kalk des Tricalcols gut ausgenutzt wird. Die positive Bilanz des $\mathrm{CaO}$-Umsatzes selbst lässt sich leicht aus dem fortwährenden Kalkmangel, unter dem das erwachsene Tier in der dieser zweiten Periode vorhergehenden Zeit infolge seines Futters stand, erklären. Aus der Tabelle II, welche die Resorptionsverhältnisse 
für das Tricalcol darstellt, ergibt sich, dass Kalk zı ca. $69 \%$ der angewandten Menge' resorbiert wurde; die Phosphorsäure blieb etwas hinter diesem Werte zurück. Jedenfalls ergeben diese Zahlen, dass im Dünndarm Tricalciumphosphat in kolloidaler Form in Gegenwart von Eiweisskörpern in beträchtlichem Maasse resorbierbar ist, was bezüglich des Kalkes eine weitere Bestätigung der früheren Versuche an Kaninchen und Hund bedeutet.

I) Bilanz in Tabelle III (Periode II) ergibt eine Anlagerung von $+0,6861 \mathrm{~g}$ Phosphorsäure. Gegenüber der Periode $\mathrm{I}$, die ein Minus von - $0,3905 \mathrm{~g} \mathrm{P}_{2} \mathrm{O}_{5}$ aufwies, ist dies also eine Verbesserung $\mathrm{um}+1,0766 \mathrm{~g} \mathrm{P}_{2} \mathrm{O}_{5}$, was beinahe genau der Zufubr der Trieaicolphosphorsäure dureh die Vella-Fistel $(1,0671 \mathrm{~g})$ entsprieht. Es ergibt sich hieraus, dass die durch die Vella-Fistel zugeführte Phosphorsäure vollständig ausgenutzt wurde. Auch in Periode II spricht das Verhältnis zwischen Kotkalk und Kotphosphorsäure für die Ausscheifung des Kalkes als Tricalciumphosphat.

In Periode II sind also die Bilanzen des $\mathrm{CaO}$ und $\mathrm{P}_{2} \mathrm{O}_{5}$, die in Periode I negativ waren, infolge Zufuhr von Tricalcol durch die isolierte Dünndarmschlinge in positive Bilanzen umgewandelt worden. Diese Feststellungen sind im Sinne der Kochmann'schen Arbeit, in der durch Zulage von $\mathrm{Ca}_{3}\left(\mathrm{PO}_{4}\right)_{2}$ zur Nahrung positive Kalkbilanz erzielt wird. Allerdings wird bei vorliegenden Versuchen auch die Phosphorsäurebilanz eine positive, während dieselbe bei Kochmann III. Mitteilung Versuch II, meist negativ (trotz teilweise hohen $\mathrm{N}$-Ansatzes) bleibt. $\mathrm{Ob}$ dieses Verhalten im Zusammenhange mit der Art des verwendeten Präparates steht, lässt sich ohne weiteres nicht entscheiden.

Wäbrend dieser zweiten Periode verlor das Tier $100 \mathrm{~g}$ Gewicht, war jedoch stets völlig munter. Um nun ganz sicher bei den Feststellungen zu sein, wurden die beiden Perioden wiederholt, jedoch zur Erzie]ung einer positiven N-Bilanz mit dem Unterscbiede, dass ein Drittel Nahrung mehr gereicht wurde. Auch zu diesen Versuchen diente die gleiche Nahrung wie bei Periode I und II. Die Dauer der folgenden Versuche war wiederum 6 Tage; sie verliefen, ohne dass Fehler vorkamen. Der Kot war gut abzutrennen. (Siehe Tab. IV auf S. 240.)

Wir sehen in diesem Versuche die Einwirkung der Nahrungsvermehrung durch den Ansatz von $3,7708 \mathrm{~g} \mathrm{~N}$ in der Periode, während die Kalkbilanz entsprechend den Feststellungen Kochmann's 
Tabelle IV.

Periode III. 6 Tage. Futter viermal $490 \mathrm{~g}$ sterilisiertes Pferdefieisch.

\begin{tabular}{l|c|c|c|c|c|c|c}
\hline & \multicolumn{2}{|c|}{$\begin{array}{c}\text { Einnahme } \\
\mathrm{g}\end{array}$} & $\begin{array}{c}\text { Gesamt- } \\
\text { einnahme } \\
\text { in der } \\
\text { Periode }\end{array}$ & \multicolumn{2}{|c|}{$\begin{array}{c}\text { Ausgabe } \\
\mathrm{g}\end{array}$} & $\begin{array}{c}\text { Gesamt- } \\
\text { ausgabe } \\
\text { in der } \\
\text { Periode }\end{array}$ & $\begin{array}{c}\text { Bilanz } \\
\mathrm{g}\end{array}$ \\
\cline { 2 - 6 } & Futter & $\begin{array}{l}\text { kalloidal. } \\
\text { Kalkpräp. }\end{array}$ & Kot & & \\
\hline $\mathrm{N} .$. & 59,9760 & 0 & 59,9760 & 58,7643 & 2,4409 & 56,2052 & $+3,7708$ \\
$\mathrm{CaO}$. & 0,4940 & 0 & 0,4940 & 0,2318 & 0,5072 & 0,7390 & $-0,2450$ \\
$\mathrm{P}_{2} \mathrm{O}_{5}$ & 7,1188 & 0 & 7,1188 & 5,6757 & 0,3842 & 6,0599 & $+1,0589$
\end{tabular}

Kotgewicht: lufttrocken $31,9646 \mathrm{~g}$.

negativ wird. Es verlangt also die zugelegte Nahrung eine gewisse Kalkmenge. Die Phosphorsäurebilanz ist dagegen positiv $+1,0589 \mathrm{~g}$, was durch die grössere $\mathrm{P}_{2} \mathrm{O}_{5}$-Zufuhr und die positive N-Bilanz dieser Periode erklärlich ist. Die Kotzahlen für Kalk und Phosphorsäure machen die Ausscheidung als $\mathrm{Ca}_{3}\left(\mathrm{PO}_{4}\right)_{2}$ wahrscheinlich. Die Verdauung des Nahrungsstickstoffes ist eine vorzügliche, ungefähr $95,9 \%$.

In der folgenden Periode IV wurde die gleiche Nahrung verabreicht, jedoch dem Hunde durch die isolierte Darmschlinge täglich in gleicher Weise wie bei Periode II $3 g$ Tricalcol zugeführt. Sämtliche bei Periode II beschriebenen Versuchsmaassregeln wurden auch hier angewandt. Diese Versuchsabteilung dauerte 6 Tage, in denen 6 mal $3 \mathrm{~g}=18 \mathrm{~g}$ Tricalcol in die Vella-Fistel eingegeben wurden. In der gesammelten Spülfüssigkeit aus der V ell a - Fistel wurden Kalk und Phosphorsäure bestimmt. Tabelle V zeigt die Resorption während dieser Periode.

$$
\text { Tabelle V. }
$$

Aufnahme des Tricalcols durch die isolierte Darmschlinge in Periode IV.

\begin{tabular}{|c|c|c|c|c|}
\hline & $\begin{array}{c}\text { Eingegeben } \\
\mathbf{g}\end{array}$ & $\begin{array}{l}\text { Zurück- } \\
\text { analysiert } \\
\mathrm{g}\end{array}$ & $\begin{array}{c}\text { Resorbiert } \\
\mathbf{g}\end{array}$ & $\begin{array}{l}\text { Resorption } \\
\text { in Prozenten } \\
\text { der Eingabe }\end{array}$ \\
\hline $\begin{array}{l}\mathrm{N} \\
\mathrm{CaO}\end{array}$ & $\begin{array}{l}1,8540 \\
1,9764 \\
1,6110\end{array}$ & $\begin{array}{c}\text { (geschätzt) } \\
0,4610 \\
0,3864\end{array}$ & $\begin{array}{c}(1,4215) \\
1,5154 \\
1,2246\end{array}$ & $\begin{array}{l}(76,67) \\
76,67 \\
76,01\end{array}$ \\
\hline
\end{tabular}

Auch hier wurde wieder der grösste Teil des kolloidalen Kalkpräparates resorbiert, und zwar stieg die Resorption des Kalkes von $69,01 \%$ in Periode II auf $76,67 \%$. Auch die Phosphorsäure zeigt eine vermehrte Resorption, die bis auf eine Kleinigkeit mit der Theorie für $\mathrm{Ca}_{3}\left(\mathrm{PO}_{4}\right)_{2}$ übereinstimmt, so dass auch durch diesen 
Versuch wieder die Aufnahme des Kalkes und der Phosphorsäure als kolloidales Tricalciumphosphat durch den Dünndarm festgestellt wurde. Die von der isolierten Darmschlinge resorbierten Mengen Kalk und Phosphorsäure sind bedeutend und entsprechen einer täglichen Aufnahme von ungefähr $0,5 \mathrm{~g} \mathrm{Ca}_{3}\left(\mathrm{PO}_{4}\right)_{2}$. Den während dieses Versuches durchgeführten Stoffwechselversuch schildert die folgende Tabelle VI.

$$
\text { Tabelle VI. }
$$

Periode IV. 6 Tage. Futter: viermal $490 \mathrm{~g}$ sterilisiertes Pferdefleisch, hierzu $18 \mathrm{~g}$ Tricalcol durch die isolierte Darmschlinge.

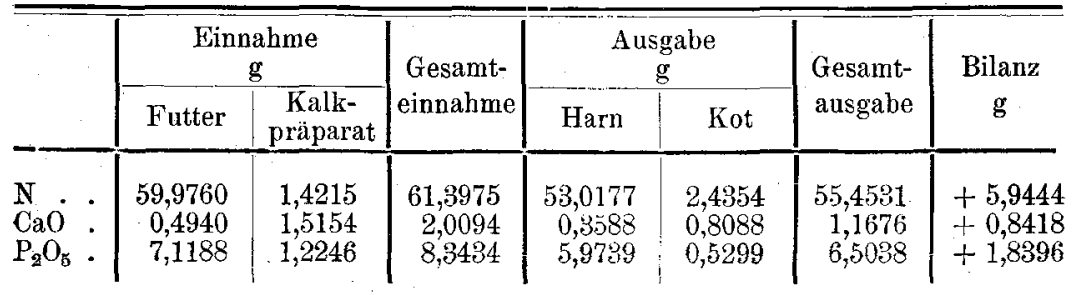

Kotgewicht: lufttrocken $31,7040 \mathrm{~g}$.

Die in Periode III durch vernehrtes Futter auf $+3,7708 \mathrm{~g} \mathrm{~N}$ gewachsene Stickstoffbilanz steigt in Periode IV auf $+5,9444 \mathrm{~g} \mathrm{~N}$; der Stickstoff ist zu $96 \%$ verwertet. Wir finden hier eine Vermehrung des Stickstoffansatzes über die Zulage durch das Tricalcol hinaus; es hat also eine etwas bessere Verwertung des Stickstoffes stattgefunden. Schon in Periode II ist auffällig, dass der durch das Tricalcol zugefuhrte Stickstoff vollständig ausgenutzt wurde, und tritt dies in Periode IV bei ungefähr gleicher Zulage noch mehr hervor. Es drängt sich dabei der Gedanke auf, dass diese auffällig gute Stickstoffausnutzung im Zusammenhange mit der Kalkaufnahme steht.

Den Einfluss von Kalk und Phosphorsäure auf die Stickstoffbilanz behandelt Hagemann, Bonn, in seinem kürzlich im Arch. f. d. ges. Physiol. Bd. 146 ersehienenen „Beitrag zur Wirkung der Mineralsubstanzen im Tierkörper" und gelangt dabei zu folgendem Resultat:

„Aus den vorstehend aufgeführten Untersuchungen möchte ich nun den Schluss ziehen, dass beim wachsenden Hammel der Reichtum der Nahrung an Mineralsubstanzen, vornehmlich an Kalk und Phosphorsäure, eine sehr grosse Rolle spielt, so dass bei einem Mangel an diesen sogar die Assimilation der stickstoff haltigen Substanzen erheblich leidet; es kommt aber auch auf die Art der 
Vermengung resp. Verteilung der Mineralsubstanz im Futter an derart, dass eine sebr feine Verteilung, wie sie z. B. durch den Finkler'schen Vermahlungsprozess gewährleistet wird, oder wie sie durch das natüriche Verwachsensein in an Mineralsubstanz reichen Futtermitteln gegeben ist, ganz beträchtlich wirksamer wie eine grobe Vermengung ist. Inwieweit diese Schlüsse auch bei anderen pflanzenfressenden Haustieren Gültigkeit haben, müssen weitere Versuche erweisen."

Die bessere Ausnutzung des Stickstoffes bei Kalkzulage bedarf in meinem Falle noch weiterer Bestätigung. Sollten diese Verhältnisse sich bei weiteren Versuchen bestätigen, so könnte mancher pathologisehe Zustand Erklärung finden, und würde sich ein günstiger Ausblick auf rationelle Eruährung und Therapie eröffnen.

Im Vergleiche zu Periode III ist die Kalkbilanz in Periode IV gestiegen, und zwar ist sie von $-0,2450 \mathrm{~g}$ auf $+0,8418 \mathrm{~g}$ gewachsen. Auch in diesem Falle finden wir den vom Dünndarm resorbierten Kalk bestens ausgenützt. Vergleichen wir jedoch die Kalkbilanz der Periode II mit der von Periode IV, so finden wir die von II $(+1,0305 \mathrm{~g} \mathrm{CaO})$ um $0,1887 \mathrm{~g}$ höher als die von Periode IV $(+0,8418 \mathrm{~g} \mathrm{CaO})$. Trotzdem nun in Periode IV ein Drittel Nahrung. mehr verabreicht wurde als in II und trotz erhöhter Aufnahme vou $\mathrm{CaO}$, sowohl per os als auch durch den isolierten Darmteil, ist die Kalkbilanz doch weniger positiv als in Periode II geworden, so dass hier eine weitere Bestätigung der von $\mathrm{Kochmann}$ festgestellten Abhängigkeit der Kalkbilanz von der Nahrungszufuhr vorliegt. Die in Periode IV verabreichte Nahrung verlangt nach den in Periode III gemachten Erfahrungen einen Verlust von $-0,2450 \mathrm{~g} \mathrm{CaO}$; Periode IV zeigt eine Bilanz von $+0,8418 \mathrm{~g} \mathrm{CaO}$, es sind also $+1,0868 \mathrm{~g} \mathrm{CaO}$ aus der Tricalcolzulage gedeckt worden, was einer Verwertung von ca. $71,7 \%$ entspricht.

Was nun den Phosphorsäureumsatz anbelangt, so sind auch hier die Verhältnisse ähnlich wie in den Periodes I und II. Wir fiuden die Phosphorsäurebilanz wesentlich von $+1,0589$ in Periode III auf $+1,8396 \mathrm{~g}$ in Periode IV gestiegen, was sich sowohl aus dem Kalkals dem Stickstoffansatze erklärt.

Nachfolgende Tabellen VII, VIII und IX zeigen Stickstoff-, Kalk- und Phosphorsäurestoffwechsel zur besseren Übersicht für die vier Versuchsperioden vereinigt und gleichzeitig die Verteilung der Ausscheidungen durch Harn und Kot. 
Beitrag zur Aufnahme und Verwertung vou Kalk und Phosphorsäure etc. 243

Tabelle VII. Stickstoff.

\begin{tabular}{|c|c|c|c|c|c|c|c|c|}
\hline \multirow{2}{*}{ Versuch } & \multirow{2}{*}{ Tage } & \multicolumn{2}{|c|}{$\underset{\mathrm{g} N}{\text { Einnahme }}$} & \multicolumn{2}{|c|}{$\begin{array}{c}\text { Ausgabe } \\
\mathrm{g} N\end{array}$} & \multicolumn{2}{|c|}{$\begin{array}{l}\text { Verteilung der } \\
\text { Gesamtausgabe }\end{array}$} & \multirow{2}{*}{$\underset{\mathrm{g} \mathrm{N}}{\mathrm{Bilanz}}$} \\
\hline & & Futter & $\begin{array}{c}\text { Tri- } \\
\text { calcol }\end{array}$ & Harn & Kot & Harn & Kot & \\
\hline \begin{tabular}{rr}
\multicolumn{3}{c}{ Periode I } \\
& II \\
$"$ & III \\
& IV
\end{tabular} & $\begin{array}{l}6 \\
6 \\
6 \\
6\end{array}$ & $\begin{array}{l}44,9820 \\
44,9820 \\
59,9760 \\
59,9760\end{array}$ & $\begin{array}{c}0 \\
1,27,94 \\
0 \\
1,4215\end{array}$ & $\begin{array}{l}44,8603 \\
44,9168 \\
53,7643 \\
53,0177\end{array}$ & $\begin{array}{l}2,0595 \\
2,2048 \\
2,4409 \\
2,4354\end{array}$ & $\begin{array}{l}95,65 \% \\
95,11 \% \\
95,66 \% \\
95,61 \%\end{array}$ & $\begin{array}{l}4,35 \% \\
4,89 \% \\
4,34 \% \\
4,39 \%\end{array}$ & $\begin{array}{r}-1,9178 \\
-0,8602 \\
+3,7708 \\
+5,9444\end{array}$ \\
\hline
\end{tabular}

Tabelle VIII. Kalk.

\begin{tabular}{|c|c|c|c|c|c|c|c|c|}
\hline \multirow{2}{*}{ Versuch } & \multirow{2}{*}{ Tage } & \multicolumn{2}{|c|}{$\underset{\mathrm{g} \mathrm{CaO}}{\text { Einnabme }}$} & \multicolumn{2}{|c|}{$\begin{array}{c}\text { Ausgabe } \\
\text { g CaO }\end{array}$} & \multicolumn{2}{|c|}{$\begin{array}{l}\text { Verteilung der } \\
\text { Gesamtausgabe }\end{array}$} & \multirow{2}{*}{$\begin{array}{l}\text { Bilanz } \\
\text { g CaO }\end{array}$} \\
\hline & & Futter & $\begin{array}{c}\text { Tri- } \\
\text { calcol }\end{array}$ & Harn & Kot & Harn & Kot & \\
\hline 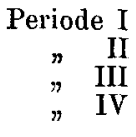 & $\begin{array}{l}6 \\
6 \\
6 \\
6\end{array}$ & $\begin{array}{l}0,3705 \\
0,3705 \\
0,4940 \\
0,4940\end{array}$ & $\begin{array}{c}0 \\
1,3639 \\
0 \\
1,5154\end{array}$ & $\begin{array}{l}0,1868 \\
0,2760 \\
0,2318 \\
0,3588\end{array}$ & $\begin{array}{l}0,3273 \\
0,4279 \\
0,5072 \\
0,8088\end{array}$ & $\begin{array}{l}36,34 \% \\
39,21 \% \\
31,36 \% \\
30,73 \%\end{array}$ & $\begin{array}{r}63,66 \% \\
60,79 \% \\
68,64 \% \\
69,27 \%\end{array}$ & $\begin{array}{r}-0,1436 \\
+1,0305 \\
+0,2450 \\
+0,8418\end{array}$ \\
\hline
\end{tabular}

Tabelle IX. Phosphorsäure.

\begin{tabular}{|c|c|c|c|c|c|c|c|c|}
\hline \multirow{2}{*}{ Versuch } & \multirow{2}{*}{ Tage } & \multicolumn{2}{|c|}{$\underset{\text { g } P_{2} \mathrm{O}_{5}}{\text { Einnahme }}$} & \multicolumn{2}{|c|}{$\begin{array}{l}\text { Ausgabe } \\
\mathrm{g} \mathrm{P}_{2} \mathrm{O}_{5}\end{array}$} & \multicolumn{2}{|c|}{$\begin{array}{l}\text { Verteilnng der } \\
\text { Gesamtausgabe }\end{array}$} & \multirow{2}{*}{$\begin{array}{l}\text { Bilanz } \\
\text { g } \mathrm{P}_{2} \mathrm{O}_{5}\end{array}$} \\
\hline & & Futter & $\begin{array}{l}\text { Tri- } \\
\text { calcol }\end{array}$ & Harn & Kot & Harn & Kot & \\
\hline \begin{tabular}{cc}
\multicolumn{2}{c}{ Periode I } \\
$\Rightarrow$ & II \\
$"$ & III \\
$"$ & IV
\end{tabular} & $\begin{array}{l}6 \\
6 \\
6 \\
6\end{array}$ & $\begin{array}{l}5,3391 \\
5,3391 \\
7,1188 \\
7,1188\end{array}$ & $\begin{array}{c}0 \\
1,0671 \\
0 \\
1,2246\end{array}$ & $\begin{array}{l}5,4294 \\
5,3459 \\
5,6757 \\
5,9739\end{array}$ & $\begin{array}{l}0,3002 \\
0,3742 \\
0,3842 \\
0,5299\end{array}$ & $\begin{array}{l}94,76 \% \\
93,47 \% \\
93,66 \% \\
91,85 \%\end{array}$ & $\begin{array}{l}5,24 \% \\
6,53 \% \\
6,34 \% \\
8,15 \%\end{array}$ & $\begin{array}{r}-0,3905 \\
+0,6861 \\
+1,0589 \\
+1,8396\end{array}$ \\
\hline
\end{tabular}

Tabelle X ergibt die Gesamtbilanz der Versuchsperioden I und III ohne Kalkzulage zusammen, die Tabelle XI die Gesamtbilanzen der Perioden II und IV mit Zulage des Tricalcols zusammen.

T a belle X. Periode I und III.

\begin{tabular}{|c|c|c|c|}
\hline & $\begin{array}{c}\text { Gesamteinnabme } \\
\mathrm{g}\end{array}$ & $\begin{array}{c}\text { Gesamtausgabe } \\
\mathrm{g}\end{array}$ & $\begin{array}{l}\text { Bilanz } \\
\mathrm{g}\end{array}$ \\
\hline $\begin{array}{l}\mathrm{N} \\
\mathrm{CaO} \\
\mathrm{P}_{2} \mathrm{O}_{5}\end{array}$ & $\begin{array}{r}104,9580 \\
0,8645 \\
12,4579\end{array}$ & $\begin{array}{r}103,1050 \\
1,2531 \\
11,7895\end{array}$ & $\begin{array}{l}+1,8530 \\
+0,3986 \\
+0,6684\end{array}$ \\
\hline
\end{tabular}


Tabelle XI. Periode II und IV.

\begin{tabular}{|c|c|c|c|}
\hline & $\begin{array}{c}\text { Gesamteinnahme } \\
\mathrm{g}\end{array}$ & $\begin{array}{c}\text { Gesamtausgabe } \\
\mathrm{g}\end{array}$ & $\begin{array}{l}\text { Bilanz } \\
\text { g }\end{array}$ \\
\hline 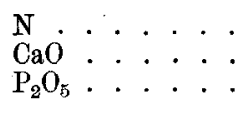 & $\begin{array}{r}107,6589 \\
3,7438 \\
14,7496\end{array}$ & $\begin{array}{r}102,5747 \\
1,8715 \\
12,2239\end{array}$ & $\begin{array}{l}+5,0842 \\
+1,8723 \\
+2,5257\end{array}$ \\
\hline
\end{tabular}

Vergleichen wir diese beiden Tabellen miteinander, so finden wir bezüglich der N-Bilanz ein wesentliches Anwachsen bei den Kalkperioden, sogar über die N-Zulage durch das Präparat hinaus. Die Tatsache stimmt mit den erwähnten Untersuchungen $\mathrm{Hage-}$ mann's, Bonn, an Hammeln bei Verabreichung verschieden zubereiteter kalkphosphathaltiger Nahrung überein.

In den Perioden ohne Kalkzulage (Tab. X) haben wir eine negative $\mathrm{CaO}-\mathrm{Bilanz}$, während die Kalkzulageperioden (Tab. XI) eine wesentlich positive Bilanz aufweisen. In Tabelle $\mathrm{X}$ zeigt der Phosphorstoffwechsel nur ein geringes Plus, während in den Kalkzulageperioden Tabelle XI die Bilanz stark positiv geworden ist. Diese positive $\mathrm{P}_{2} \mathrm{O}_{5}$ - Bilanz zeigt unter Berücksichtigung der Anlagerung von $\mathrm{CaO}$ und Stickstoff, die ungefähr $1,7 \mathrm{~g}$ bzw. $0,7 \mathrm{~g} \mathrm{P}_{2} \mathrm{O}_{5}$ erfordern, gute Übereinstimmung mit der Theorie. Weiter bestätigen diese Gesamtbilanzen, dass Kalk und Phosphorsäure des durch den isolierten Darmteil aufgenommenen kolloidalen Tricalciumphosphatkaseins (Tricalcol) in hohem Masse zur Verwertung resp. zur Anlagerung kommen.

Zur Vervollständigung der vorstehenden Versuche mit dem alkalilöslichen kolloidalen Tricalciumphosphatkasein schien es mir geboten, an dem gleichen Tiere die Einwirkung einer Zulage von gewöhnlichem Tricalciumphosphat zur Nahrung zu untersuchen. Zu diesem Zwecke wurden die Stoffwechselversuche in gleicher Weise weitergeführt; das Tier erhielt in zwei Perioden die bisher übliche Fleischnahrung, während in einer dritten Periode eine Zulage von gewöhnlichem Tricalciumphosphat zu der Nahrung verabreicht wurde. In der ersten Periode bekam das Tier während 6 Tagen die gleiche Nahrung wie in Periode IV; die folgende Tabelle XII gibt über den Versuch Aufschluss.

Wir finden in dieser Periode eine vorzügliche Ausnützung des Nahrungsstickstoffes und einen wesentlichen Ansatz von Stickstoff, der noch grösser ist als in den Perioden III und IV, in denen das 
Beitrag zur Aufnahme und Verwertung von Kalk und Phosphorsäure etc. 245

T abelle XII.

Periode V. 6 Tage. Futter: viermal $490 \mathrm{~g}$ sterilisiertes Pferdefleisch.

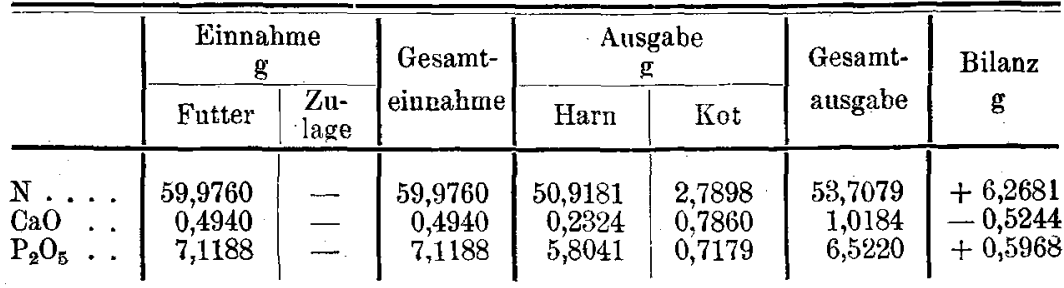

gleiche Futter verabreicht wurde. Betrachten wir die Kalkbilanz, so finden wir einen Verlust von $-0,5244 \mathrm{~g} \mathrm{CaO}$, also wesentlich mehr als in der korrespondierenden Periode III, die bei gleicher Fütterung nur eine Unterbilanz von $-0,2450 \mathrm{~g} \mathrm{CaO}$ aufwies. Die Phosphorsäurebilanz ist mit $+0,5968 \mathrm{~g}$ wesentlich niedriger als in Periode III $(+1,0589 \mathrm{~g})$, was sich wohl zum Teil aus der stärker negativen Kalkbilanz erklären lässt. Im Kote finden sich Kalk und Phosphorsäure in Mengen, die für deren Ausscheidung als Tricalciumphosphat sprechen.

Zur Fortsetzung der Versuche musste eine neue Menge Pferdefleisch sterilisiert werden. Die Sterilisation wurde in Portionen von $350 \mathrm{~g}$ vorgenommen; es wurden ausgesuchte magere Stücke Pferdefleisch in feingehacktem Zustande verwendet. Die Analysen ergaben folgende Zahlen: $\mathrm{N} \mathrm{3,083 \% ,} \mathrm{CaO} 0,0201 \%, \mathrm{P}_{2} \mathrm{O}_{5} \quad 0,4349 \%$.

Um etwaige Einflüsse des Futterwechsels auszuschalten, wählte ich für die folgende Periode eine Dauer von 9 Tagen. Die Nahrungsmenge war mit $350 \mathrm{~g}$ Fleiseh pro Tag so bemessen, das sie möglichst wenig von den in den vorhergehenden Versuchsperioden verabreichten Futtermengen abwich. Der Verlauf dieses Vẹrsuches ergibt sich aus Tabelle XIII.

Tabelle XIII.

Periode VI. 9 Tage. Futter: neunmal $350 \mathrm{~g}$ sterilisiertes Pferdefleisch.

\begin{tabular}{|c|c|c|c|c|c|c|c|}
\hline & \multicolumn{2}{|c|}{$\begin{array}{c}\text { Einnahme } \\
g\end{array}$} & \multirow{2}{*}{$\begin{array}{l}\text { Gesamt- } \\
\text { einnahme }\end{array}$} & \multicolumn{2}{|c|}{$\begin{array}{c}\text { Ausgabe } \\
\mathrm{g}\end{array}$} & \multirow{2}{*}{$\begin{array}{l}\text { Gesamt- } \\
\text { ausgabe }\end{array}$} & \multirow{2}{*}{$\begin{array}{c}\text { Bilanz } \\
\mathrm{g}\end{array}$} \\
\hline & Futter & $\begin{array}{l}\text { Zu- } \\
\text { lage }\end{array}$ & & Harn & Kot & & \\
\hline $\begin{array}{l}\mathrm{N} \\
\mathrm{CaO} \\
\mathrm{P}_{2} \mathrm{O}_{5}\end{array}$ & $\begin{array}{r}97,1145 \\
0,6331 \\
13,6993\end{array}$ & - & $\begin{array}{r}97,1145 \\
0,63331 \\
13,6993\end{array}$ & $\begin{array}{r}96,6370 \\
0,3247 \\
11,9749\end{array}$ & $\begin{array}{l}2,0518 \\
0,6846 \\
1,1041\end{array}$ & $\begin{array}{r}98,6888 \\
1,0093 \\
13,0790\end{array}$ & $\begin{array}{r}-1,5743 \\
-0,3762 \\
+0,6203\end{array}$ \\
\hline
\end{tabular}


Die Stickstoff bilanz zeigt in dieser Periode einen negativen Wert, trotzdem mehr Stickstoff pro Tag verfüttert war, als in den Perioden I bis V. Es ist dies vielleicht dadurch zu erklären, dass nur ganz mageres Fleisch verwendet worden war. Auch die Kalkbilanz ist negativ, und war dies nach den in den Perioden I, III und V gemachten Erfahrungen zu erwarten. Die Phosphorsäurebilanz dagegen ist positiv; hierfür eine Erklärung zu geben, ist mir nicht möglich. Auffällig ist auch, dass die Menge der Kotphosphorsäure wesentlich grösser ist, als dem Kotkalke entspricht.

In der nächsten Periode VII wurde an den ersten 5 Tagen eine Zulage von je $0,5 \mathrm{~g}$ feingemahlenem Tricalciumphosphat dem Futter sorgfältig beigemischt. Leider stellte sich vor Beginn der Periode heraus, dass das Futter zum Teil verdorben war; es wurde deshalb eine neue Portion ausgewähltes, mageres Pferdefleisch gehackt und in Portionen von $350 \mathrm{~g}$ sterilisiert. Dieses Futter zeigte folgende Analyse: N 3,112\%, $\mathrm{CaO} 0,0213 \%, \mathrm{P}_{2} \mathrm{O}_{5} \quad 0,4252 \%$.

Der Untersehied in den Futteranalysen der Perioden VI und VII ist so gering, dass derselbe kaum eine Rolle spielen kann. Nachfolgende Tabelle XIV zeigt die Verhältnisse bei diesem Stoffwechselversuch.

Tabelle XIV.

Periode VII. 9 Tage. Futter: Neunmal $350 \mathrm{~g}$ sterilisiertes Pferdefleisch; hierzu fünfmal je $0,5=2,5 \mathrm{~g}$ fein gemahlenes Tricalciumphosphat.

\begin{tabular}{|c|c|c|c|c|c|c|c|}
\hline & \multicolumn{2}{|c|}{$\begin{array}{c}\text { Einnahme } \\
g\end{array}$} & \multirow{2}{*}{$\begin{array}{c}\text { Gesamt- } \\
\text { einnahme }\end{array}$} & \multicolumn{2}{|c|}{$\begin{array}{c}\text { Ausgabe } \\
\mathrm{g}\end{array}$} & \multirow{2}{*}{$\begin{array}{l}\text { Gesamt- } \\
\text { ausgabe }\end{array}$} & \multirow{2}{*}{$\begin{array}{c}\text { Bilanz } \\
\text { g }\end{array}$} \\
\hline & Futter & Zulage & & Harn & Kot & & \\
\hline $\begin{array}{l}\mathrm{N} \\
\mathrm{CaO} \\
\mathrm{P}_{2} \mathrm{O}_{5}\end{array}$ & $\begin{array}{r}98,0280 \\
0,6709 \\
13,3938\end{array}$ & $\begin{array}{l}\overline{1,2502} \\
1,0107\end{array}$ & $\begin{array}{r}98,0280 \\
1,9211 \\
\mathbf{1 4 , 4 0 4 5}\end{array}$ & $\begin{array}{r}94,2985 \\
0,3156 \\
12,1087\end{array}$ & $\begin{array}{l}3,4859 \\
1,5447 \\
1,8885\end{array}$ & $\begin{array}{r}97,7844 \\
1,8603 \\
13,9972\end{array}$ & $\begin{array}{r}+0,2436 \\
+0,0608 \\
+0,4073\end{array}$ \\
\hline
\end{tabular}

In dieser Periode ist die Stickstoffbilanz schwach positiv $=$ $+0,2436 \mathrm{~g} \mathrm{~N}$, während sie bei nahezu gleichem Futter in Periode VI - die N-Differenz ist nur $0,9 \mathrm{~g}$ - einen negativen Wert von - $1.5743 \mathrm{~g} \mathrm{~N}$ zeigte. Auch hier müssen wir wieder die Einwirkung der Kalkzulage auf die Stickstoffbilanz zur Erk]ärung für diese Erscheinung heranziehen, um so mehr, als eine Verschiedenheit in der Zusammensetzung der Nahrung - die Fleischanalysen stimmen nahezu überein - nicht angenommen werden kann. Die Kalkbilanz ist in dieser Periode schwach positiv geworden. Die Zulage von gewöhnlichem Tricalciumphosphat zum Futter hat also die negative 
Bilanz der vorhergehenden Periode in eine positive verwandelt. Während in Periode VI die Kalkbilanz ein Minus von $0,3762 \mathrm{~g}$ $\mathrm{CaO}$ aufweist, zeigt Periode VII ein Plus von $+0,0608 \mathrm{~g} \mathrm{CaO}$, was eine Steigerung von $+0,4370 \mathrm{~g} \mathrm{CaO}$ bedeutet. Diese Kalkmenge ist also durch die Zulage des Tricalciumphosphates zum Futter gedeckt worden. Die verfütterten $2,5 \quad \mathrm{~g} \quad \mathrm{Ca}_{3}\left(\mathrm{PO}_{4}\right)_{2}$ enthielten $1,2502 \mathrm{~g} \mathrm{CaO}$, von denen $0,4370 \mathrm{~g} \mathrm{CaO}$ verwertet wurden, was einer Ausnutzung von ungefähr $35 \%$ entspricht. Betrachtet man die Ausscheidungszahlen für Kalk in Periode VII, so findet man bezüglich des Harnkalkes nichts Bemerkenswertes gegenüber der vorhergehenden Periode; dagegen ist der Kotkalk sehr wesentlich gestiegen, und zwar noch über die in der Kalkzulage enthaltene Menge hinaus. Vergleichen wir diesen Befund mit den Zablen der Perioden II und IV, in denen wir durch den Dünndarm alkalilösliches kolloidales Tricalciumphosphat zuführten, so sehen wir, dass in letzteren Perioden wohl auch die Menge des Kotkalkes gestiegen ist, aber bei weitem nicht in dem Maasse wie bei der Verabreichung von gewöhnlichem Kalkphosphat. Die Kalkbilanzen der Perioden II und IV zeigen eine wesentliche Rentention bei Verwendung des kolloidalen $\mathrm{Ca}_{3}\left(\mathrm{PO}_{4}\right)_{2}$ des Tricalcols, während die Kalkbilanz der Periode VII bei Verabreichung des gewöhnlichen Tricalciumphosphates per os nur eine minimale Retention aufweist. Für Tricalcolkalk fanden wir eine Verwertung von ungefähr $86 \%$ in Periode II und $71 \%$ in Periode IV, während wir für den Kalk des gewöhnlichen Tricalciumphosphates in Periode VII nur eine Verwertung von $35 \%$ feststellen konnten.

Die Phosphorsäurebilanz zeigt in Periode VII keine Besonderheiten; sie ist jedoch niedriger als in Periode VI.

Die Feststellungen der Perioden VI und VII sind um so bemerkenswerter, als sie uns zeigen, dass die Verwertung des gewöhnlichen Tricalciumphosphates eine geringe ist im Vergleiche mit dem alkalilöslichen kolloidalen $\mathrm{Ca}_{3}\left(\mathrm{PO}_{4}\right)_{2}$ des Tricalcols. Auch diese Versuche bestätigten den schon so oft erbrachten Nachweis, dass Kalkphosphat einen günstigen Einfluss auf die Kalkbilanz hat; wie weit jedoch der Einfluss in unserem Falle reicht, sehen wir beim Vergleich von Periode VI und VII. Es wurde in Periode VII allerdings die negative Kalkbilanz gedeckt, aber zu einer wesentlichen Retention, d. h. zum Ersatz der in den Vorperioden verloren gegangenen Kalkmengen kam es nicht. Auffällig ist dabei, dass die 
durch den Kot ausgeschiedenen Mengen Kalk und Phosphorsäure in Periode VII sehr gross sind, und drängt sich hier die Frage auf, ob dies vielleicht von einer unvollstänaligen Resorption des gewöhnlichen Tricalciumphosphates herruhrt. In den Perioden II und IV haben wir die Ausscheidungsverbältnisse von kolloidalem $\mathrm{Ca}_{3}\left(\mathrm{PO}_{4}\right)_{2}$, das durch den Dünndarm resorbiert war, kennen gelernt; wir finden dort beim Vergleich mit Periode I und III wohl auch eine erhöhte Ausscheidung von Kalkphosphat durch den Kot, aber bei weitem nicht in dem Maasse, wie sie bei Periode VII im Vergleich zu Periode VI vorliegt. Diese Tatsache macht es wahrscheinlich, dass das gewöhnliche Tricalciumphosphat den Magen zum Teil unverändert passiert bat und so in den Dünndarm gelangt ist; dass der Dünndarm aber gewöhnliches $\mathrm{CaO}_{3}\left(\mathrm{PO}_{4}\right)_{2}$ nicht zu resorbieren vermag, haben wir in den vorstehenden Versuchen festgestellt. Somit scheint es möglich, dass die schlechtere Ausnutzung des Kalkphosphates mit der Magentätigkeit bzw. Magensäureproduktion im Zusammenhange steht, und sind unsere weiteren Arbeiten darauf gerichtet, die Rolle, die der Magen bei der Verwertung des Kalkphosphates spielt, aufzuklären.

Da wir eingangs festgestellt hatten, dass vom isolierten Dünndarm gewöhnliches Tricalciumphosphat nicht aufgenommen, während das kolloidale Kalkphosphat des Tricalcols gut resorbiert wird, lag der Gedanke nahe, auch andere calciumphosphathaltige Körper zur Untersuchung heranzuziehen; hierzu bot die Milch das grösste Interesse.

Die erwähnte Verschiedenheit in der Fällbarkeit der Kuhmilch gegenüber Frauenmilch und einer Lösung des Tricalcols durch Natronlauge bei einer Konzentration von Normalfünftellauge liess auch Unterschiede bezüglich ihrer Resorbierbarkeit im Darme erwarten. Nachdem durch die Resorptionsversuche festgestellt war, dass alkalilösliches kolloidales Tricalciumphosphatkasein (Tricalcol) in bedeutendem Maasse vom Dünndarm aufgenommen wird, schien es geboten, die ausgeführten Resorptionsversuche im Vergleiche mit Kuhmilch zu wiederholen. Frauenmilch zu diesen Versuchen heranzuziehen, war wegen ihres geringen Kalkgehaltes wenig aussichtsvoll, wenn nicht relativ grosse Mengen in Anwendung gebracht wurden. Dieses verbot jedoch die Versuchsordnung von selbst; denn erstens liessen sich nach in Vorversuchen gemachten Erfahrungen nur Mengen von ca. $50 \mathrm{ccm}$ im Zeitraume von 2-6 Stunden in den isolierten Darmteil einbringen, ohne dass durch die Ausgangsfistel 
Material dem Versuch entzogen wurde, und zweitens hätte die gesamte Versuchsdauer bei der einige Aussicht auf Frfolg bietenden Menge von 200 cem Frauenmilch auf das ungefähr Vierfache, also 24 Stunden, erhöht werden müssen, was wohl kaum durchführbar gewesen wäre. Diese Versuche wurden deshalb auf Kuhmilch und eine alkalische Lösung des Tricalcols besehränkt. Um die Verbältnisse möglichst gleich zu gestalten, wurden $50 \mathrm{ccm}$ nahezu fettfreier, zentrifugierter Kuhmilch verwendet mit einem Zusatz von $0,05 \mathrm{~g}$. Soda. Letzteres aus dem Grunde, weil dem Tricalcol, von dem $1 \mathrm{~g}$ zur Anwendung gelangte, die gleiche Menge Soda in $50 \mathrm{ccm}$ Wasser zur Lösung zugesetzt werden musste. Zum Ausgleich des Zuckergehaltes wurden der Kalkpräparatlösung 2,5 g Milchzucker zugegeben. Die Versuche wurden an aufeinanderfolgenden Tagen an dem im Stoffwechselkäfig befindlichen Hunde mit Vella-Fistel, der dauernd, mit der gleichen Nahrung wie bei den im vorstehenden geschilderten Stoffwechselversuchen gefüttert, und dessen Darmschlinge täglich in gleicher Weise ausgespült wurde, ausgefübrt.

Nachdem das Tier frühmorgens die Hälfte seiner Futterration erhalten hatte, wurde es angeschirrt und in der aus der Abbildung ersichtlichen Weise auf das Gerüst befestigt. Dann wurde die VellaFistel mit $250 \mathrm{ccm}$ körperwarmer, kalkfreier, physiologischer Kochsalzlösung, die einen Zusatz von $0,1 \%$ Natriumkarbonat hatte, ausgespült und diese Flüssigkeit analysiert. Der eigentliche Versuch begann ca. 1/2 Stunde nach beendigter Ausspülung, und zwar wurde innerhalb 2 Stunden in Partien von $10 \mathrm{cem}$ die Lösung von $1 \mathrm{~g}$ Tricalcol in $50 \mathrm{ccm} 0,1 \%$ iger Sodalösung unter Zusatz von 2,5 g Milchzucker durch einen Katheter genau wie früher eingegeben. Hierauf wurden innerbalb 3 Stunden $50 \mathrm{ccm}$ pbysio[ogische Kochsalzlösung, 0,1\% Soda enthaltend, $10 \mathrm{ccm}$-weise einlaufen gelassen und nach Verlauf einer weiteren Stunde, also nach 6 Stunden Versuchszeit, der Darm mit $750 \mathrm{ccm}$ alkalischer physiologischer Kochsalzlösung ausgespült. Sämtliche aus dem Darm austretende Flüssigkeit wurde sorgfältig gesammelt und analysiert. Am bequemsten war dazu die Neumann'sche Methode nach vorherigem Eindampfen der grossen Flüssigkeitsmengen. Der Kalk wurde aus der Aschenlösung als Oxalat gefällt und als $\mathrm{CaO}$ gewogen. Die Genauigkeit der angewandten mechanischen Arbeitsweise erwiesen zwei Vorversuche mit 2 Stunden Versuchszeit, bei 
denen sämtlicher zur Verwendung gelangter Kalk der Kuhmilch bei den Analysen wiedergefunden wurde, da bei der kurzen Versuchszeit eine wesentliche Zersetzung der Milcheiweisskörper noch nicht stattgefunden batte. Offenbar verlangsamten die relativ hohe Konzentration und die Gegenwart der immerhin wesentlichen Milchzuckermengen die Aufnahme. Diese Vorversuche veranlassten auch den Zusatz des Milchzuckers zur Lösung des Tricalcols.

Am Morgen nach dem ersten Versuche mit dem Kalkpräparat wurde, wie oben erwähnt, die isolierte Darmschlinge ausgespült und in der Flüssigkeit der Kalk bestimmt. Diese Maassnahme diente zur Kontrolle für eine genügende Ausspülung am vergangenen Versuchstage. Nach der Ausspülung begann der zweite Versuch mit den oben beschriebenen entfetteten $50 \mathrm{ccm}$ Kuhmilch, denen $0,05 \mathrm{~g}$ Soda zugesetzt waren. Bei diesen Versuchen wurden peinlichst genau die Flüssigkeitsmengen und Zeiten eingehalten. Die weiter angeführten Versuche sind Kontrollversuche, und ergibt nachfolgende Tabelle XV alles Nähere:

Tabelle XV.

\begin{tabular}{|c|c|c|c|c|c|c|c|c|}
\hline \multirow[b]{2}{*}{$\mathrm{Nr}}$. & \multirow[b]{2}{*}{ Präparat } & \multirow{2}{*}{$\begin{array}{c}\text { Ver- } \\
\text { suchs- } \\
\text { zeit }\end{array}$} & \multirow{2}{*}{$\begin{array}{c}\text { Ein- } \\
\text { geguben } \\
\text { g CaO }\end{array}$} & \multirow{2}{*}{$\begin{array}{l}\text { Aus- } \\
\text { gespült } \\
\text { g } \mathrm{CaO}\end{array}$} & \multicolumn{2}{|c|}{$\begin{array}{c}\text { Resorption } \\
\text { in }\end{array}$} & \multirow{2}{*}{$\begin{array}{l}\text { In Proz. } \\
\text { der Durch- } \\
\text { schnitts- } \\
\text { aufnahme } \\
\text { des Mileb- } \\
\text { kalks }\end{array}$} & \multirow{2}{*}{$\begin{array}{c}\text { Ausgesp. } \\
\text { am } \\
\text { nächsten } \\
\text { Morgen } \\
\text { g CaO }\end{array}$} \\
\hline & & & & & $\mathrm{g} \mathrm{CaO}$ & $\begin{array}{c}\text { Proz. } \\
\text { der } \\
\text { Eingabe }\end{array}$ & & \\
\hline 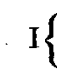 & $\begin{array}{l}\text { Tricalcol u } \\
\text { Milchzucker }\end{array}$ & 6 & 0,1098 & 0,0922 & 0,0176 & 16,02 & 198 & 0,0032 \\
\hline II & Milch & 6 & 0,0804 & 0,0710 & 0,0094 & 11,56 & 100 & 0,0028 \\
\hline III \{ & $\begin{array}{l}\text { Tricalcol u. } \\
\text { Milchzucker }\end{array}$ & 6 & 0,1098 & 0,0802 & 0,0296 & 26,95 & 334 & 0,0026 \\
\hline IV & Milch & 6 & 0,0835 & 0,0752 & 0,0083 & 9,98 & 100 & 0,0027 \\
\hline$\nabla\{$ & $\begin{array}{l}\text { Tricalcol u. } \\
\text { Milchzucker }\end{array}$ & 6 & 0,0824 & 0,0599 & 0,0225 & 27,30 & 254 & 0,0029 \\
\hline
\end{tabular}

Wir sehen also, dass bei diesen mit aller Vorsicht ausgeführten Versuchen die Menge des resorbierten Kalkes der Kuhmilch wesentlich geringer ist wie die des resorbierten Tricalcolkalkes; es wird der Kalk aus dem kolloidalen Kalkphosphatkasein durch die gleiche Dünndarmschliuge in mehr als doppelter Menge aufgenommen wie der Milchkalk.

Die bei Versuch I und III angewendeten Kalkmengen sind allerdings den Milchkalkmengen nicht ganz gleich, ein Mangel, der 
diesen Versuchen zum Vorwurf gemacht werden könnte, wenn nicht auch die Resorptionszahlen in Prozenten des angewandten $\mathrm{CaO}$ eine deutliche Sprache redeten. Die Wiederholung der Versuche (Nr. III und IV) ergeben ein ähnliches Resultat, das zugunsten des Tricalcolkalkes verschoben ist. Versuch V ist mit einer bezüglich ihres Kalkgehaltes der Milch gleichenden Tricalcollösung ausgeführt worden und zeigt einen ähnlichen Resorptionswert wie die Versuche I und III mit grösserer Tricalcolmenge. Nachdem nun die Versuche I bis $\mathrm{V}$ der Tabelle $\mathrm{XV}$ dargetan haben, dass der Milchkalk wesentlich schwerer resorbiert wird als Tricalcolkalk, auch wenn er unter gleichzeitiger Beigabe einer wesentlichen Menge Milehzuckers angewandt wird, ist noch der Einfluss der verschieden grossen Stickstoffmenge der Milch und des Tricalcols auszuschalten. Die zu diesem Versuche (Tabelle XVI) benutzten $50 \mathrm{ccm}$ Milch wurden wie oben mit $0,05 \mathrm{~g}$ Soda versetzt, und sonst wurde in gleicher Weise wie früher gearbeitet. Zum Vergleiche wurden dagegen von dem Kalkpräparat nur $0,75 \mathrm{~g}$ angewendet, um möglichst gleichen CaO-Gehalt zu erreichen; die Zuckermenge wurde wieder zu $2,5 \mathrm{~g}$ angenommen, jedoch wurden, um einen Ausgleich bezüglich des verschiedenen Stickstoffgehaltes zu erzielen, selbst hergestellte reinste Präparate von Kasein $(1 \mathrm{~g})$ und Lactalbumin $(0,25 \mathrm{~g})$ als neutrale Natronsalze der Lösung des Tricalcols beigemischt. Es war somit alles geschehen, um ein der Milch nach Möglichkeit in $\mathrm{CaO}-, \mathrm{N}-$ und Kohlenhydratkonzentration gleiches Produkt in Anwendung zu bringen. Selbstverständlich konnte auf die geringen Mengen anderer in der. Milch enthaltenen Substanzen keine Rücksicht genommen werden; der Zweck dieser Maassnahme war ja auch nur, die in wesentlicher Menge in der Milch enthaltenen, etwa die Aufnahme des Kalkes verzögernden Substanzen zur Auwendung zu bringen. Es handelt sich ja bei den Versuchen nicht um in der Natur vorkommende Verhältnisse, sondern allein um die Feststellung, ob der Kalk der Milch in einer isolierten Darmschlinge gleich gut resorbiert wird wie der Kalk des alkalilöslichen Kalkpräparates, selbst wenn ihm die meisten in der Milch vorkommenden Körper zugesetzt sind. Das Resultat ergibt denn auch nur einen Anhaltspunkt über die Beschaffenheit des in der Kuhmilch vorhandenen Kalkes, während es bezüglich des natürlichen Verhaltens bei der Aufnahme der Milch per os nichts aussagt, fehlen doch bei diesem Versuche die Säure- 
wirkung des Magens völlig und die Fermentwirkungen des normalen Verdauungstraktus zum grössten Teile.

Nachstehende Tabelle XVI zeigt das Resultat des Versuches:

Tabelle XVI.

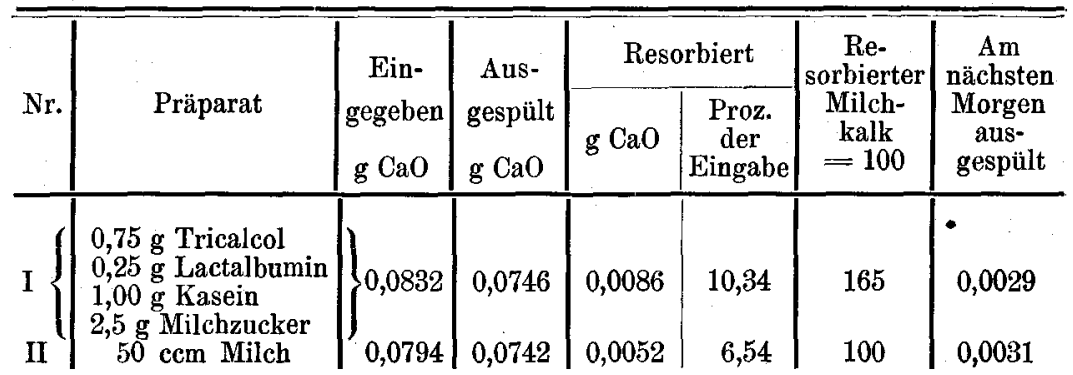

Es geht aus vorstehenden Zahlen hervor, dass in beiden Versuchen die Menge des resorbierten Kalkes wesentlich geringer ist als bei der Versuchsreihe der Tabelle XV. Die Versuche wurden an zwei aufeinanderfolgenden Tagen ausgeführt und alle Bedingungen gleich eingehalten. Jedenfalls geht auch hieraus hervor, dass der Kalk des Tricalcols bei weitem besser vom Dünndarm resorbiert wird als der Milchkalk, wenn auch der Stickstoff- und Zuckergehalt ebenso hoch waren wie in der Milch.

Es hängt also die Aufnahmefähigkeit des Kalkes nicht von in der Lösung anwesenden Kohlenhydraten und Eiweisskörpern, sondern von der Beschaffenheit des Tricalciumphosphates ab. Wahrscheinlich ist die Verschiedenheit deskolloidalen Zustandes des gelösten Tricalciumphosphates für seine Aufnahme im Dünndarme von Bedeutung.

Zeigt sich schon ein Unterschied zwischen dem in der Milch vorhandenen phosphorsauren Kalk und dem alkalilöslichen kolloidalen Tricalciumphosphat im Verhalten gegen Natronlauge, so wird diese Verschiedenheit durch die Resorptionsversuche noch weiter bewiesen. Die vorstehend gefundenen geringen absoluten Werte von einigen Zentigrammen bzw. sogar nur Milligrammen schwächen die Beweiskraft der ermittelten Resultate zweifelsohne ab; ich möchte deshalb betonen, dass diese Arbeiten und Analysen mit äusserster Vorsicht ausgeführt sind.

Überblicken wir nochmals die Ergebnisse vorstehender Untersuchungen, so finden wir: 
Beitrag zur Aufnahme und Verwertung von Kalk und Phosphorsäure etc. 253

1. aus dem kolloidalen Kalkpräparat wird Kalk von einer abgebundenen Dünndarmschlinge des Kaninchens aufgenommen, während aus gewöhnlichem Tricalciumphosphat bei Gegenwart von Kasein-Natron kein Kalk resorbiert wird. Die Wiederholung dieses Versuches ergibt das gleiche Resultat. DieVersuchszahlen sind jedoch zu klein, um bindende Schlüsse zuzulassen.

2. Versuche an einem Hunde mit Vella-Fistel ergaben eine erhebliche Aufnahme des angewandten Kalkes für das kolloidale Kalkpräparat "Tricalcol", die mit der Konzentration der angewandten Lösung schwankt. Verdünnte Lösungen scheinen höhere Resorptionswerte $z$ u ergeben wie konzentriertere. Für gewöhliches, feinst zerriebenes Tricalciumphosphat konnte eine Aufnahme nicht festgestellt werden, auch nicht bei Gegenwartvon Kasein-Natron.

3. Die an dem gleichen Hunde angestellten Stoffwechselversuche mit gleichzeitiger Verabreichung von Tricalcol durch die isolierte Darmschlinge ergaben, dass aus dem Kalkpräparat $69-76 \%$ des vorhandenen Tricalciumphosphates aufgenommen wird. Die in den kalkfreien Perioden negative Kalkbilanz wird in den Kalkpräparatperioden positiv, und zwar wird im Mittel ungefähr drei Viertel des von der isolierten Darmschlinge aufgenommenen Kalkes angesetzt. Sehr ähnlich verhält sich die Phosphorsäure, auch sie gelangt in den Tricalcolperiodenzum Ansatze. Ferner wird nicht nur der gesamte Stickstoff des durch den isolierten Darmteil aufgenommenen Präparates ausgenutzt, sondern es findet in Periode IV noch ein Ansatz über die Zulage des Kalkpräparates hinaus statt.

Vielleicht findet bei positiver CaO-Bilanz eine bessere Ausnutzung des Stickstoffes statt. Die Reihe der Stoffwechselversuche bestätigt den von Kochmann festgestellten schädigenden Einfluss der Nahrungszulage auf die Kalkbilanz. 
Die vergleichenden St offwechselversuche mit und ohne Zulage von Tricalciumphosphat zum Futter - Periode VI und VII - zeigen, dass dieses nur zu ungefähr $35 \%$ verwertet wird. Es findet trotz reichlich dargebotenen Kalkphosphates nur eine geringe Retention von Kalk statt, dagegen zeigen sich im Kote grosse Mengen von Kalk und Phosphorsäure. Ob dieselben von unverändertem Tricalciumposphat herrühren, ist nicht zu entscheiden, jedoch spricht die Unverwertbarkeit des gewöhnlichem Tricalciumphosphates im Dünndarme gegenüber der vorzüglichen Resorbierbarkeit des alkalilöslichen kolloidalen Tricalciumphosphates des Tricalcols dafür, dass das per os eingegebene gewöhnliche Tricalciumphosphat den Magen zum grösseren Teile unverändert passierte und sich so im Dunndarmeder Resorption entzog.

4. Die vergleichenden Resorptionsversuche mit Milch und milchähnlich gemachter Lösung des kolloidalen Kalkpräparates ergaben, dass Milchkalk weniger gut vom Darme resorbiert wird als der Kalk des Präparates, also vielleicht ein Unterschied in der kolloidalen Beschaffenheit des in beiden vorhandenen Tricalciumphosphates besteht, der sich auchim Verhalten gegen Alkalilauge zeigt.

Die Feststellung, dass der Dünndarm des Hundes kolloidales Tricalciumphosphat bei Gegenwart von Eiweisskörpern, also in Form des Tricalcols, ohne vorherige Magenpassage in hohem Maasse aufunehmen imstande ist, führt zu der Überlegung, dass bei der Verwertung des Nahrungskalkes ähnliche kolloidale Kalkphosphate eine Rollespielenkönnen; um so mehr, als es dem Dünndarm des Hundes, wie die Versuche zeigten, nicht möglich ist, unlösliches Tricalciumphosphat aufzunehmen.

Durch die Magensäure wird den Nahrungsmitteln Kalk entzogen, es bilden sich saure Kalkphosphate, Chlorcalcium und wohl auch Phosphorsäure in Gegenwart von Eiweissabbaprodukten. Es liegt nahe, 
dass im Dünndarm, sobald genügend Alkali vorhanden ist, diese gelösten Kalksalze bzw. Phosphatewieder in Tricalciumphosphat übergeführt werden, welches sich in Gegenwart von Eiweissspaltprodukten, die als Schutzkolloide wirken, nicht abscheidet, sondern kolloidal gelöst bleibt. Diese Auffassung ist um so berechtigter, als sie mit den Darstellungsmethoden des kolloidalen Kalkpräparates in innigstem $\mathrm{Zu}$ sammenhange steht. Unter diesen Umständen müsste von der Auflösung des Nahrungskalkes im Magen, das ist von der Menge der Magensalzsäure, die Resorption des Nabrungskalkes abhängig sein, da nur diejenige Menge Kalk und Phosphorsäure der Nahrung im Darme kolloid gelöst bleiben und dadurch resorbiertwerden könnte, die im Magen durch die Säure in Lösung gegangen war.

Versuche, die den Einfluss der Magentätigkeit auf den Kalkstoffwechsel klarstellen sollen, sind in die Wege geleitet, und behalte ich mir vor, baldmöglichst darüber zu berichten.

Sind Aufnahme und Ansatz des kolloidalen Kalkpräparates "Tricalcol" beim Menschen derbejm Hunde festgestelten ähnlich, so kommt demselben für die Kalkmedikation eine Bedeutung zu, vor allem für Fälle, bei denen ein gewisser Mangel an Magensäure vorhanden ist; gerade dann wird das Kalkphosphat der Nahrung nicht gelöst undim Darmenichtinkolloidales Produkt umgewandelt werden können. Beim Feblen der Magensalzsäure kann z. B. das Kalkphosphat der Milch nicht in ein vollkommen kolloidales Produkt übergeführt werden, und es können Verhältnisse eintreten, wie sie bei den Vergleichsversuchen von Milch mit Tricalcol in der isolierten Darmschlinge des Hundes vorliegen. Inwieweit dieser Ausblick das Richtige trifft, müssen weitere Untersuchungen zeigen. Eines jedoch hat diese Arbeit ergeben: die reichliche Aufnahme und gute Verwertung des alkalilöslichen kolloidalen Tricalciumphosphates im Dünndarme, 
256 F. Zuckmayer: Beitrag zur Aufnahme und Verwertung von Kalk etc.

eine Feststellung, die von neuem die Wichtigkeit nicht nur organiseher, sondern auch anorganischer Kolloide für den Organismus dartut.

Es ist mir eine angenehme Pflicht, auch an dieser Stelle meinem hochverehrten Lehrer, Herrn Professor Dr. Hagemann in Bonn, für die vielfachen Anregungen und tatkräftige Unterstützung beidieser Arbeit meinen aufrichtigsten Dank aususprechen. 